\title{
STABILITY OF STEADY STATES OF THE NAVIER-STOKES-POISSON EQUATIONS WITH NON-FLAT DOPING PROFILE
}

\author{
ZHONG TAN, YANJIN WANG, AND YONG WANG
}

\begin{abstract}
We consider the stability of the steady state of the compressible Navier-StokesPoisson equations with the non-flat doping profile. We prove the global existence of classical solutions near the steady state for the large doping profile. For the small doping profile, we prove the time decay rates of the solution provided that the initial perturbation belongs to $L^{p}$ with $1 \leqslant p<3 / 2$.
\end{abstract}

\section{INTRODUCTION}

The dynamics of charged particles of one carrier type (e.g., electrons) can be described by the compressible Navier-Stokes-Poisson equations:

$$
\left\{\begin{array}{l}
\partial_{t} \rho+\operatorname{div}(\rho u)=0, \\
\partial_{t}(\rho u)+\operatorname{div}(\rho u \otimes u)+\nabla p(\rho)-\mu \Delta u-\left(\mu+\mu^{\prime}\right) \nabla \operatorname{div} u=\rho \nabla \phi, \\
\Delta \phi=\rho-b, \\
\left.(\rho, u)\right|_{t=0}=\left(\rho_{0}, u_{0}\right) .
\end{array}\right.
$$

Here $\rho=\rho(t, x), u=u(t, x)$ represent the density and velocity functions of the electrons respectively, at time $t \geqslant 0$ and position $x \in \mathbb{R}^{3}$. The pressure $p=p(\rho)$ is a smooth function with $p^{\prime}(\rho)>0$ for $\rho>0$. We assume that the constant viscosity coefficients $\mu$ and $\mu^{\prime}$ satisfy the usual physical conditions

$$
\mu>0, \quad \mu^{\prime}+\frac{2}{3} \mu \geqslant 0 .
$$

The self-consistent electric potential $\phi=\phi(t, x)$ is coupled with the density through the Poisson equation, where the function $b=b(x)$ is the doping profile for the ions. We assume that $b$ is a smooth function satisfying

$$
b(x)>0, \quad \lim _{|x| \rightarrow+\infty} b(x)=\bar{b}>0 .
$$

For the pressure law $p(\rho)=\rho^{\gamma}$ with the adiabatic exponent $\gamma>3 / 2$, the global existence of weak solutions was obtained by [8] when the spatial dimension is three in the framework of Lions-Feireisl for the compressible Navier-Stokes equations [9, 3. This result was later extended by [15] to the case $\gamma>1$ when the dimension is two, where the authors introduced an idea to overcome the new difficulty caused by that the Poisson term $\rho \nabla \phi$ may not be integrable when $\gamma$ is close to one. The large-time behavior of weak solutions towards the steady state was also considered in [15. When the doping profile is flat, i.e., $b(x)=\bar{b}$, the steady state of (1.1) is the trivial constant one $(\bar{\rho}, 0,0)$ with $\bar{\rho}=\bar{b}$. The unique global solution around this constant state in $H^{N}$ was proved by [10] in the framework of Matsumura-Nishida for the Navier-Stokes

2010 Mathematics Subject Classification. 35M10; 35Q60; 35Q35.

Key words and phrases. Navier-Stokes-Poisson equations; Stability; Energy method; Time decay.

Corresponding author: Yong Wang, wangyongxmu@163.com.

Z. Tan was supported by the National Natural Science Foundation of China (No. 11271305). Y. J. Wang was supported by the National Natural Science Foundation of China (No. 11201389), the Natural Science Foundation of Fujian Province of China (No. 2012J05011), the Specialized Research Fund for the Doctoral program of Higher Education (No. 20120121120023), and the Fundamental Research Funds for the Central Universities (No. 2013121002). 
equations [11. Moreover, when the initial perturbation $\left(\rho_{0}-\bar{\rho}, u_{0}\right)$ is small in $L^{1}$, the $L^{2}$ decay of the solution to (1.1) was also obtained in [10]:

$$
\|(\rho-\bar{\rho})(t)\|_{L^{2}} \lesssim(1+t)^{-\frac{3}{4}} \text { and }\|u(t)\|_{L^{2}} \lesssim(1+t)^{-\frac{1}{4}} .
$$

This may imply that the presence of the electric field slows down the time decay rate of the velocity with the factor $1 / 2$ compared to the Navier-Stokes equations [12, 13]. It was proved by transforming the system (1.1) into the Navier-Stokes equations with a non-local force

$$
\left\{\begin{array}{l}
\partial_{t} \rho+\operatorname{div}(\rho u)=0 \\
\partial_{t}(\rho u)+\operatorname{div}(\rho u \otimes u)+\nabla p(\rho)-\mu \Delta u-\left(\mu+\mu^{\prime}\right) \nabla \operatorname{div} u=\rho \nabla \Delta^{-1}(\rho-\bar{\rho}) .
\end{array}\right.
$$

However, the author in [16] gave a different (contrary) comprehension of the effect of the electric field on the time decay rates of the solution. When the initial perturbation $\left(\rho_{0}-\bar{\rho}, u_{0}, \nabla \phi_{0}\right)$ belongs to $L^{p}$ with $1<p \leqslant 2$, the $L^{2}$ decay of the solution to (1.1) was obtained in [16]:

$$
\|(\rho-\bar{\rho})(t)\|_{L^{2}} \lesssim(1+t)^{-\frac{3}{2}\left(\frac{1}{p}-\frac{1}{2}\right)-\frac{1}{2}} \text { and }\|u(t)\|_{L^{2}} \lesssim(1+t)^{-\frac{3}{2}\left(\frac{1}{p}-\frac{1}{2}\right)} .
$$

In this sense, the electric field enhances the time decay rate of the density with the factor $1 / 2$ ! This can be understood well from the physical point of view since we get an additional dispersive effect from the repulsive electric force.

In this paper, we will study the asymptotic stability of the steady state of the system (1.1) with the non-flat doping profile $b(x)$. A steady state $\left(\rho_{s}, \phi_{s}\right)$ with $u_{s} \equiv 0$ of (1.1) must satisfy

$$
\left\{\begin{array}{l}
\nabla p\left(\rho_{s}\right)=\rho_{s} \nabla \phi_{s} \\
\Delta \phi_{s}=\rho_{s}-b .
\end{array}\right.
$$

We will record the existence and uniqueness of the solution to (1.7) in Proposition 2.1.

Notations. We use $L^{p}\left(\mathbb{R}^{3}\right), 1 \leqslant p \leqslant \infty$ to denote the $L^{p}$ spaces with norm $\|\cdot\|_{L^{p}}$, and $W^{k, p}\left(\mathbb{R}^{3}\right)$ to denote the usual Sobolev spaces with norm $\|\cdot\|_{W^{k, p}}$, and $H^{k}=W^{k, 2}$. $\nabla^{\ell}$ with $\ell \in \mathbb{R}$ stands for the usual spatial derivatives of order $\ell$; we allow that $\ell<0$ or $\ell$ is not a positive integer.

Throughout this paper, we let $C$ denote the universal positive constants. We will use $A \lesssim B$ if $A \leqslant C B$ and $A \gtrsim B$ if $A \geqslant C B$, and we may write $\frac{d}{d t} A+B \lesssim D$ for $\frac{d}{d t} A+C B \lesssim D$. For simplicity, we write $\|(A, B)\|_{X}:=\|A\|_{X}+\|B\|_{X}$ and $\int f:=\int_{\mathbb{R}^{3}} f d x$.

Our first main result of the global solutions to (1.1) near the steady state for the large doping profile is stated as the following theorem.

Theorem 1.1. Assume that $\nabla b(x) \in H^{k}$ with $k \geqslant 3$, and $\left(\rho_{s}, \phi_{s}\right)$ of (1.7) is constructed in Proposition 2.1. If $\left\|\left(\rho_{0}-\rho_{s}, u_{0}\right)\right\|_{H^{k}}+\left\|\nabla^{-1}\left(\rho_{0}-\rho_{s}\right)\right\|_{L^{2}}$ is sufficiently small, then there exists a unique global solution $(\rho, u, \nabla \phi)$ to the system (1.1) such that for all $t \geqslant 0$,

$$
\begin{aligned}
& \left\|\left(\rho-\rho_{s}, u\right)(t)\right\|_{H^{k}}^{2}+\left\|\left(\nabla \phi-\nabla \phi_{s}\right)(t)\right\|_{L^{2}}^{2}+\int_{0}^{t}\left(\left\|\left(\rho-\rho_{s}\right)(\tau)\right\|_{H^{k}}^{2}+\|\nabla u(\tau)\|_{H^{k}}^{2}\right) d \tau \\
& \quad \leqslant C\left(\left\|\left(\rho_{0}-\rho_{s}, u_{0}\right)\right\|_{H^{k}}^{2}+\left\|\nabla^{-1}\left(\rho_{0}-\rho_{s}\right)\right\|_{L^{2}}^{2}\right) .
\end{aligned}
$$

Remark 1.2. In Theorem 1.1, the initial condition on the smallness of $\left\|\nabla^{-1}\left(\rho_{0}-\rho_{s}\right)\right\|_{L^{2}}$ is required so that the initial potential energy $\left\|\nabla \phi_{0}-\nabla \phi_{s}\right\|_{L^{2}}$ is small. Indeed, since $\phi-\phi_{s}$ satisfies the Poisson equation $\Delta\left(\phi-\phi_{s}\right)=\rho-\rho_{s}$, we have

$$
\left\|\nabla \phi_{0}-\nabla \phi_{s}\right\|_{L^{2}}=\left\|\nabla \Delta^{-1}\left(\rho_{0}-\rho_{s}\right)\right\|_{L^{2}}=\left\|\nabla^{-1}\left(\rho_{0}-\rho_{s}\right)\right\|_{L^{2}} .
$$

Such condition can be guaranteed by that, for instance, $\left\|\rho_{0}-\rho_{s}\right\|_{L^{6 / 5}}$ is small.

Our second main result of the time decay rates of the solution to (1.1) towards the steady state for the small doping profile is stated as the next theorem. 
Theorem 1.3. Assume that the assumptions of Theorem 1.1 hold for $k \geqslant 4$. If $\|\nabla b\|_{H^{k}}+$ $\|b-\bar{b}\|_{L^{r}}$ with $1<r<3 / 2$ is sufficiently small and $\left\|\left(\nabla^{-1}\left(\rho_{0}-\rho_{s}\right), u_{0}\right)\right\|_{L^{p}}$ with $1 \leqslant p<3 / 2$ is finite, then for $0 \leqslant \ell \leqslant 1 / 2$,

$$
\left\|\nabla^{\ell}\left(\rho-\rho_{s}\right)(t)\right\|_{H^{k-\ell}} \leqslant C_{0}(1+t)^{-\frac{3}{2}\left(\frac{1}{\max \{p, r\}}-\frac{1}{2}\right)-\frac{\ell}{2}-\frac{1}{2}}
$$

and for $0 \leqslant \ell \leqslant 3 / 2$,

$$
\left\|\nabla^{\ell} u(t)\right\|_{H^{k-\ell}} \leqslant C_{0}(1+t)^{-\frac{3}{2}\left(\frac{1}{\max \{p, r\}}-\frac{1}{2}\right)-\frac{\ell}{2}}
$$

and

$$
\left\|\left(\rho-\rho_{s}, u\right)(t)\right\|_{L^{\infty}} \leqslant C_{0}(1+t)^{-\frac{3}{2}\left(\frac{1}{\max \{p, r\}}-\frac{1}{2}\right)-\frac{3}{4}},
$$

where $C_{0}$ is a positive constant depending on the initial data.

Remark 1.4. In Theorem [1.3, the initial condition on the boundedness of $\left\|\nabla^{-1}\left(\rho_{0}-\rho_{s}\right)\right\|_{L^{p}}$ with $1 \leqslant p<3 / 2$ is required. If $p>1$, then such condition can be guaranteed by that, for instance, $\rho_{0}-\rho_{s}=\operatorname{div} f$ for some $f \in L^{p}$, thanks to the singular integral theory [14].

Remark 1.5. Theorems 1.1 and 1.3 extend the previous results of [16]. Note that the argument of proving the time decay of the solution in [16], which follows a pure energy method introduced in [5], highly depends on that the doping profile is flat. To show the time decay of the solution for the non-flat doping profile, we shall need to employ a different argument as explained below.

Remark 1.6. In [1, 2], the authors proved the time decay of the compressible Navier-Stokes equations with an external potential force provided that the initial perturbation belongs to $L^{p}$ with $1 \leqslant p<6 / 5$. We may expect to employ our arguments of proving Theorem 1.3 to extend the range of $p$ therein to be $1 \leqslant p<3 / 2$. The key point is to introduce the fractional derivatives in the study of the time decay as already seen from (1.9)-(1.10).

Theorems 1.1 and 1.3 will be proved in Sections 34, respectively. To prove Theorem 1.1, we will reformulate the system (1.1) into (3.5) for the perturbation $\varrho=\rho-\rho_{s}$ and $u=u$. To derive the energy estimates, the difficulty is caused by the terms on the left-hand side of (3.5). More precisely, we can not directly control as in [6] the terms resulting from when the differential operator $\nabla^{l}$ commutates with the functions of $\rho_{s}$ since $\rho_{s}$ may not be close to a positive constant in the current case. To overcome this difficulty, we first notice that these commutator terms do not appear when $l=0$, which allows us to derive the zero-order energy estimates as stated in Lemma 3.1. When $l=1,2, \ldots, k$, we will carry out the delicate analysis so that we can control these commutator terms with an (large) error term as stated in Lemma 3.2. briefly speaking, $\|\nabla u\|_{L^{2}}$. However, this error has been controlled by the previous step. Hence, after recovering the dissipation estimates of $\varrho$ by Lemmas $3.3 \sqrt{3.4}$, we can close the energy estimates.

To prove Theorem 1.3, we will reformulate (3.5) into the system (4.1) with constant coefficients. Since $\rho_{s}$ is close to $\bar{\rho}$, we can improve the energy estimates in the proof of Theorem 1.1 to deduce the estimates (4.59), which implies that the decay of $\left\|\nabla^{\ell-1} \varrho(t)\right\|_{H^{k+1-\ell}}^{2}+\left\|\nabla^{\ell} u(t)\right\|_{H^{k-\ell}}^{2}$ can be obtained from the decay of $\left\|\nabla^{\ell-1} \varrho(t)\right\|_{L^{2}}^{2}+\left\|\nabla^{\ell} u(t)\right\|_{L^{2}}^{2}+\|(\varrho, u)(t)\|_{L^{\infty}}^{2}$ for $0 \leqslant \ell \leqslant 3 / 2$. On the other hand, using the linear decay estimates of the linear Navier-Stokes-Poisson equations with constant coefficients, we can derive the estimates conversely. This interplay would then be closed by the smallness of the solution and the doping profile. Finally, we may remark that if we do not introduce the fractional derivatives, then we can only choose $\ell=0,1$ in (4.59), which would result that we could only prove Theorem 1.3 for $1 \leqslant p<6 / 5$.

The rest of this paper is organized as follows. In Section 2, we will prove the existence of the stationary solution to (1.7). We will prove Theorem 1.1 and Theorem 1.3 in Section 3 and Section 4, respectively. Some analytic tools will be collected in Appendix. 


\section{Steady STAte}

In this section, we record the following existence and uniqueness of the solutions to (1.7).

Proposition 2.1. Assume that $b(x)$ is a smooth function satisfying (1.3). Then there exists a unique classical solution $\left(\rho_{s}, \phi_{s}\right)$ to (1.7). Moreover,

- $\rho_{s}$ has the positive upper and lower bounds, i.e.,

$$
0<\inf _{x \in \mathbb{R}^{3}} b(x) \leqslant \rho_{s}(x) \leqslant \sup _{x \in \mathbb{R}^{3}} b(x)<\infty ;
$$

- if $\nabla b \in H^{k}$ with $k \geqslant 3$, then there exists a constant $C$ depending on $\|\nabla b\|_{H^{k}}$ such that

$$
\left\|\nabla \rho_{s}\right\|_{H^{k}} \leqslant C
$$

- if $\|\nabla b\|_{H^{k}}$ is sufficiently small, then

$$
\left\|\nabla \rho_{s}\right\|_{H^{k}} \lesssim\|\nabla b\|_{H^{k}}
$$

- if further $\|b-\bar{b}\|_{L^{r}}<\infty$ with $1<r<\infty$, then

$$
\left\|\rho_{s}-\bar{\rho}\right\|_{W^{2, r}} \lesssim\|b-\bar{b}\|_{L^{r}},
$$

where $\bar{\rho}=\bar{b}$.

Proof. The existence and uniqueness of the classical solutions to (1.7) satisfying the first three assertions were proved in [6] for $k=3$, but the case $k \geqslant 4$ can be handled in the same way and so we omit the proof. We may then focus on proving the last assertion a priori.

To this end, setting $h^{\prime}(s)=p^{\prime}(s) / s$, we deduce from (1.7) that

$$
\operatorname{div}\left(h^{\prime}\left(\rho_{s}\right) \nabla \rho_{s}\right)=\rho_{s}-b .
$$

Writing $f=\rho_{s}-\bar{\rho}$ with $\bar{\rho}=\bar{b}$, we rewrite (2.5) as

$$
-h^{\prime}(\bar{\rho}) \Delta f+f=\operatorname{div}\left(\left(h^{\prime}\left(\rho_{s}\right)-h^{\prime}(\bar{\rho})\right) \nabla f\right)+b-\bar{b} .
$$

It then follows from the standard elliptic theory [14] on (2.6) that for $1<r<\infty$,

$$
\begin{aligned}
\|f\|_{W^{2, r}} & \lesssim\left\|\operatorname{div}\left(\left(h^{\prime}\left(\rho_{s}\right)-h^{\prime}(\bar{\rho})\right) \nabla f\right)+b-\bar{b}\right\|_{L^{r}} \\
& \lesssim\left\|\nabla \rho_{s}\right\|_{H^{k}}\|f\|_{W^{2, r}}+\|b-\bar{b}\|_{L^{r}} .
\end{aligned}
$$

This implies (2.4) since $\left\|\nabla \rho_{s}\right\|_{H^{k}}$ is small by (2.3).

\section{Global solution with large doping profile}

In this section, we will construct the global solutions near the steady state to (1.1) for the large doping profile. For this, we define the perturbation by

$$
\varrho=\rho-\rho_{s}, u=u, \Phi=\phi-\phi_{s} .
$$

In order to reformulate the problem (1.1) properly, we introduce the enthalpy function

$$
h(z)=\int_{1}^{z} \frac{p^{\prime}(s)}{s} d s .
$$

We also introduce the Taylor expansion

$$
h\left(\varrho+\rho_{s}\right)=h\left(\rho_{s}\right)+h^{\prime}\left(\rho_{s}\right) \varrho+\mathcal{R},
$$

where the remainder $\mathcal{R}$ is given by

$$
\mathcal{R}=\int_{\rho_{s}}^{\varrho+\rho_{s}} h^{\prime \prime}(s)\left(\varrho+\rho_{s}-s\right) d s .
$$


Then the problem (1.1) can be reformulated into the perturbed form of

$$
\left\{\begin{array}{l}
\partial_{t} \varrho+\operatorname{div}\left(\rho_{s} u\right)=-\operatorname{div}(\varrho u), \\
\partial_{t} u+\nabla\left(h^{\prime}\left(\rho_{s}\right) \varrho\right)-\frac{1}{\rho_{s}}\left(\mu \Delta u+\left(\mu+\mu^{\prime}\right) \nabla \operatorname{div} u\right)-\nabla \Phi \\
\quad=-u \cdot \nabla u-\nabla \mathcal{R}+\left(\frac{1}{\varrho+\rho_{s}}-\frac{1}{\rho_{s}}\right)\left(\mu \Delta u+\left(\mu+\mu^{\prime}\right) \nabla \operatorname{div} u\right) \\
\Delta \Phi=\varrho \\
\left.(\varrho, u)\right|_{t=0}=\left(\varrho_{0}, u_{0}\right) .
\end{array}\right.
$$

3.1. Energy estimates. In this subsection, we will derive the a priori estimates for the solutions to the Navier-Stokes-Poisson equations (3.5) by assuming that for sufficiently small $\delta>0$,

$$
\|(\varrho, u)(t)\|_{H^{k}}+\|\nabla \Phi(t)\|_{L^{2}} \leqslant \delta
$$

We first derive the zero-order energy estimates for the solution itself.

Lemma 3.1. It holds that

$$
\frac{d}{d t} \int\left(h^{\prime}\left(\rho_{s}\right) \varrho^{2}+\rho_{s}|u|^{2}+|\nabla \Phi|^{2}\right)+\|\nabla u\|_{L^{2}}^{2} \lesssim \delta\|\varrho\|_{L^{2}}^{2} .
$$

Proof. Multiplying the first two equations in (3.5) by $h^{\prime}\left(\rho_{s}\right) \varrho$ and $\rho_{s} u$ respectively, summing up them and then integrating over $\mathbb{R}^{3}$, we obtain

$$
\begin{aligned}
\frac{1}{2} \frac{d}{d t} \int\left(h^{\prime}\left(\rho_{s}\right) \varrho^{2}+\rho_{s}|u|^{2}\right)+\mu\|\nabla u\|_{L^{2}}^{2}+\left(\mu+\mu^{\prime}\right)\|\operatorname{div} u\|_{L^{2}}^{2}-\int \rho_{s} u \cdot \nabla \Phi \\
=-\int\left(h^{\prime}\left(\rho_{s}\right) \varrho \operatorname{div}(\varrho u)+\rho_{s} u \cdot(u \cdot \nabla u)+\rho_{s} u \cdot \nabla \mathcal{R}\right) \\
\quad+\int \rho_{s} u \cdot\left(\frac{1}{\varrho+\rho_{s}}-\frac{1}{\rho_{s}}\right)\left(\mu \Delta u+\left(\mu+\mu^{\prime}\right) \nabla \operatorname{div} u\right) .
\end{aligned}
$$

Here we have used the integration by parts to have the cancelation:

$$
\int\left(h^{\prime}\left(\rho_{s}\right) \varrho \operatorname{div}\left(\rho_{s} u\right)+\rho_{s} u \cdot \nabla\left(h^{\prime}\left(\rho_{s}\right) \varrho\right)\right)=0 .
$$

To estimate the Poisson term on the left-hand side of (3.8), we integrate by parts by several times, use the first equation and the Poisson equation in (3.5), by Hölder's and Sobolev's inequalities and the a priori bound (3.6), and employ the standard elliptic estimates $\left\|\nabla^{k} \Phi\right\|_{L^{2}} \lesssim$ $\left\|\nabla^{k-2} \varrho\right\|_{L^{2}}$ for $k \geqslant 2$, to deduce

$$
\begin{aligned}
-\int \rho_{s} u \cdot \nabla \Phi & =\int \operatorname{div}\left(\rho_{s} u\right) \Phi=-\int \partial_{t} \varrho \Phi-\int \operatorname{div}(\varrho u) \Phi \\
& =-\int \partial_{t} \Delta \Phi \Phi+\int \varrho u \cdot \nabla \Phi=\frac{1}{2} \frac{d}{d t} \int|\nabla \Phi|^{2}+\int \varrho u \cdot \nabla \Phi \\
& \geqslant \frac{1}{2} \frac{d}{d t} \int|\nabla \Phi|^{2}-C\|\varrho\|_{L^{2}}\|u\|_{L^{3}}\|\nabla \Phi\|_{L^{6}} \\
& \geqslant \frac{1}{2} \frac{d}{d t} \int|\nabla \Phi|^{2}-C \delta\|\varrho\|_{L^{2}}^{2} .
\end{aligned}
$$

We now estimate the terms on the right-hand side of (3.8). By Hölder's and Sobolev's inequalities, (3.6) and (2.1), we obtain

$$
\begin{aligned}
& -\int\left(h^{\prime}\left(\rho_{s}\right) \varrho \operatorname{div}(\varrho u)+\rho_{s} u \cdot(u \cdot \nabla u)\right) \\
& +\int \rho_{s} u \cdot\left(\frac{1}{\varrho+\rho_{s}}-\frac{1}{\rho_{s}}\right)\left(\mu \Delta u+\left(\mu+\mu^{\prime}\right) \nabla \operatorname{div} u\right) \\
& \lesssim\|\varrho\|_{L^{2}}\|\nabla \varrho\|_{L^{3}}\|u\|_{L^{6}}+\|\varrho\|_{L^{2}}\|\varrho\|_{L^{\infty}}\|\operatorname{div} u\|_{L^{2}} \\
& \quad+\|u\|_{L^{6}}\|u\|_{L^{3}}\|\nabla u\|_{L^{2}}+\|u\|_{L^{6}}\|\varrho\|_{L^{2}}\left\|\nabla^{2} u\right\|_{L^{3}}
\end{aligned}
$$




$$
\lesssim \delta\left(\|\varrho\|_{L^{2}}^{2}+\|\nabla u\|_{L^{2}}^{2}\right) .
$$

For the remaining term involved with $\nabla \mathcal{R}$, we integrate by parts to have

$$
\begin{aligned}
-\int \rho_{s} u \cdot \nabla \mathcal{R} & =\int \nabla \rho_{s} \cdot u \mathcal{R}+\rho_{s} \operatorname{div} u \mathcal{R} \\
& \lesssim\|u\|_{L^{\infty}}\|\varrho\|_{L^{2}}^{2}+\|\operatorname{div} u\|_{L^{2}}\|\varrho\|_{L^{2}}\|\varrho\|_{L^{\infty}} \\
& \lesssim \delta\left(\|\varrho\|_{L^{2}}^{2}+\|\nabla u\|_{L^{2}}^{2}\right) .
\end{aligned}
$$

Here we have used the fact from (3.3)-(3.4) that $\mathcal{R}=O\left(\varrho^{2}\right)$.

Plugging the estimates (3.10)-(3.12) into (3.8), since $\delta$ is small, we then conclude (3.7).

We then derive the energy estimates for the derivatives of the solution.

Lemma 3.2. For $l=1, \ldots, k$, we have that for any $\varepsilon>0$,

$$
\begin{aligned}
& \frac{d}{d t} \int\left(h^{\prime}\left(\rho_{s}\right)\left|\nabla^{l} \varrho\right|^{2}+\rho_{s}\left|\nabla^{l} u\right|^{2}\right)+\left\|\nabla^{l+1} u\right\|_{L^{2}}^{2} \\
& \quad \lesssim(\delta+\varepsilon)\left(\left\|\nabla^{l} \varrho\right\|_{L^{2}}^{2}+\|\varrho\|_{L^{2}}^{2}\right)+C_{\varepsilon}\left(\left\|\nabla^{l} u\right\|_{L^{2}}^{2}+\|\nabla u\|_{L^{2}}^{2}\right) .
\end{aligned}
$$

Proof. Applying $\nabla^{l}$ to the first two equations in (3.5) and then multiplying the resulting identities by $h^{\prime}\left(\rho_{s}\right) \nabla^{l} \varrho$ and $\rho_{s} \nabla^{l} u$ respectively, summing up them and then integrating over $\mathbb{R}^{3}$, we obtain

$$
\begin{aligned}
& \frac{1}{2} \frac{d}{d t} \int\left(h^{\prime}\left(\rho_{s}\right)\left|\nabla^{l} \varrho\right|^{2}+\rho_{s}\left|\nabla^{l} u\right|^{2}\right)+\int\left(h^{\prime}\left(\rho_{s}\right) \nabla^{l} \varrho \nabla^{l} \operatorname{div}\left(\rho_{s} u\right)+\rho_{s} \nabla^{l} u \cdot \nabla^{l} \nabla\left(h^{\prime}\left(\rho_{s}\right) \varrho\right)\right) \\
& -\int \rho_{s} \nabla^{l} u \cdot \nabla^{l}\left(\frac{1}{\rho_{s}}\left(\mu \Delta u+\left(\mu+\mu^{\prime}\right) \nabla \operatorname{div} u\right)\right)-\int \rho_{s} \nabla^{l} u \cdot \nabla^{l} \nabla \Phi \\
& =-\int h^{\prime}\left(\rho_{s}\right) \nabla^{l} \varrho \nabla^{l} \operatorname{div}(\varrho u)-\int \rho_{s} \nabla^{l} u \cdot \nabla^{l}(u \cdot \nabla u)-\int \rho_{s} \nabla^{l} u \cdot \nabla^{l} \nabla \mathcal{R} \\
& \quad+\int \rho_{s} \nabla^{l} u \cdot \nabla^{l}\left(\left(\frac{1}{\varrho+\rho_{s}}-\frac{1}{\rho_{s}}\right)\left(\mu \Delta u+\left(\mu+\mu^{\prime}\right) \nabla \operatorname{div} u\right)\right) .
\end{aligned}
$$

First, we estimate the terms on the left-hand side of (3.14). By integrating by parts by several times and employing the commutator notation (A.2), we have

$$
\begin{aligned}
\int & \left(h^{\prime}\left(\rho_{s}\right) \nabla^{l} \varrho \nabla^{l} \operatorname{div}\left(\rho_{s} u\right)+\rho_{s} \nabla^{l} u \cdot \nabla^{l} \nabla\left(h^{\prime}\left(\rho_{s}\right) \varrho\right)\right) \\
= & \int\left(h^{\prime}\left(\rho_{s}\right) \nabla^{l} \varrho \nabla^{l} \operatorname{div}\left(\rho_{s} u\right)-\operatorname{div}\left(\rho_{s} \nabla^{l} u\right) \nabla^{l}\left(h^{\prime}\left(\rho_{s}\right) \varrho\right)\right) \\
= & \int\left(h^{\prime}\left(\rho_{s}\right) \nabla^{l} \varrho \operatorname{div}\left(\left[\nabla^{l}, \rho_{s}\right] u\right)+\rho_{s} \nabla^{l} u \cdot \nabla\left[\nabla^{l}, h^{\prime}\left(\rho_{s}\right)\right] \varrho\right) \\
= & \int h^{\prime}\left(\rho_{s}\right) \nabla^{l} \varrho\left(\left[\nabla^{l}, \rho_{s}\right] \operatorname{div} u+\left[\nabla^{l}, \nabla \rho_{s}\right] u\right) \\
& +\int \rho_{s} \nabla^{l} u \cdot\left(\left[\nabla^{l}, h^{\prime}\left(\rho_{s}\right)\right] \nabla \varrho+\left[\nabla^{l}, \nabla h^{\prime}\left(\rho_{s}\right)\right] \varrho\right) .
\end{aligned}
$$

For the second term on the right-hand side of (3.15), we employ the commutator estimates (A.3) to have: for $l=1$,

$$
\begin{gathered}
\int h^{\prime}\left(\rho_{s}\right) \nabla \varrho\left[\nabla, \nabla \rho_{s}\right] u \lesssim\|\nabla \varrho\|_{L^{2}}\left\|\left[\nabla, \nabla \rho_{s}\right] u\right\|_{L^{2}} \\
\lesssim\|\nabla \varrho\|_{L^{2}}\left\|\nabla^{2} \rho_{s}\right\|_{L^{3}}\|u\|_{L^{6}} \lesssim\|\nabla \varrho\|_{L^{2}}\|\nabla u\|_{L^{2}}
\end{gathered}
$$

for $l \geqslant 2$,

$$
\int h^{\prime}\left(\rho_{s}\right) \nabla^{l} \varrho\left[\nabla^{l}, \nabla \rho_{s}\right] u \lesssim\left\|\nabla^{l} \varrho\right\|_{L^{2}}\left\|\left[\nabla^{l}, \nabla \rho_{s}\right] u\right\|_{L^{2}}
$$




$$
\begin{aligned}
& \lesssim\left\|\nabla^{l} \varrho\right\|_{L^{2}}\left(\left\|\nabla^{2} \rho_{s}\right\|_{L^{3}}\left\|\nabla^{l-1} u\right\|_{L^{6}}+\left\|\nabla^{l+1} \rho_{s}\right\|_{L^{2}}\|u\|_{L^{\infty}}\right) \\
& \lesssim\left\|\nabla^{l} \varrho\right\|_{L^{2}}\left(\left\|\nabla^{l} u\right\|_{L^{2}}+\|\nabla u\|_{H^{1}}\right) \lesssim\left\|\nabla^{l} \varrho\right\|_{L^{2}}\left(\left\|\nabla^{l} u\right\|_{L^{2}}+\|\nabla u\|_{L^{2}}\right) .
\end{aligned}
$$

Note that we have used (2.2) and the interpolation from Lemma A.1 $\left\|\nabla^{2} u\right\|_{L^{2}} \lesssim\left\|\nabla^{l} u\right\|_{L^{2}}+$ $\|\nabla u\|_{L^{2}}$ for $l \geqslant 2$. We may then conclude that this term can be bounded by

$$
\left\|\nabla^{l} \varrho\right\|_{L^{2}}\left(\left\|\nabla^{l} u\right\|_{L^{2}}+\|\nabla u\|_{L^{2}}\right) \text {. }
$$

We can derive the same bound for the first term that for $l=1$,

for $l \geqslant 2$,

$$
\begin{gathered}
\int h^{\prime}\left(\rho_{s}\right) \nabla \varrho\left[\nabla, \rho_{s}\right] \operatorname{div} u \lesssim\|\nabla \varrho\|_{L^{2}}\left\|\left[\nabla, \rho_{s}\right] \operatorname{div} u\right\|_{L^{2}} \\
\lesssim\|\nabla \varrho\|_{L^{2}}\left\|\nabla \rho_{s}\right\|_{L^{\infty}}\|\nabla u\|_{L^{2}} \lesssim\|\nabla \varrho\|_{L^{2}}\|\nabla u\|_{L^{2}}
\end{gathered}
$$

$$
\begin{aligned}
& \int h^{\prime}\left(\rho_{s}\right) \nabla^{l} \varrho\left[\nabla^{l}, \rho_{s}\right] \operatorname{div} u \lesssim\left\|\nabla^{l} \varrho\right\|_{L^{2}}\left\|\left[\nabla^{l}, \rho_{s}\right] \operatorname{div} u\right\|_{L^{2}} \\
& \quad \lesssim\left\|\nabla^{l} \varrho\right\|_{L^{2}}\left(\left\|\nabla \rho_{s}\right\|_{L^{\infty}}\left\|\nabla^{l-1} \operatorname{div} u\right\|_{L^{2}}+\left\|\nabla^{l} \rho_{s}\right\|_{L^{6}}\|\operatorname{div} u\|_{L^{3}}\right) \\
& \quad \lesssim\left\|\nabla^{l} \varrho\right\|_{L^{2}}\left(\left\|\nabla^{l} u\right\|_{L^{2}}+\|\nabla u\|_{H^{1}}\right) \lesssim\left\|\nabla^{l} \varrho\right\|_{L^{2}}\left(\left\|\nabla^{l} u\right\|_{L^{2}}+\|\nabla u\|_{L^{2}}\right)
\end{aligned}
$$

Similarly, for the third and fourth terms, we have: for $l=1$,

$$
\begin{aligned}
\int & \rho_{s} \nabla u \cdot\left(\left[\nabla, h^{\prime}\left(\rho_{s}\right)\right] \nabla \varrho+\left[\nabla, \nabla h^{\prime}\left(\rho_{s}\right)\right] \varrho\right) \\
& \lesssim\|\nabla u\|_{L^{2}}\left(\left\|\left[\nabla, h^{\prime}\left(\rho_{s}\right)\right] \nabla \varrho\right\|_{L^{2}}+\left\|\left[\nabla, \nabla h^{\prime}\left(\rho_{s}\right)\right] \varrho\right\|_{L^{2}}\right) \\
& \lesssim\|\nabla u\|_{L^{2}}\left(\left\|\nabla h^{\prime}\left(\rho_{s}\right)\right\|_{L^{\infty}}\|\nabla \varrho\|_{L^{2}}+\left\|\nabla^{2} h^{\prime}\left(\rho_{s}\right)\right\|_{L^{3}}\|\varrho\|_{L^{6}}\right) \lesssim\|\nabla u\|_{L^{2}}\|\nabla \varrho\|_{L^{2}}
\end{aligned}
$$

for $l \geqslant 2$,

$$
\begin{aligned}
\int \rho_{s} \nabla^{l} u \cdot\left(\left[\nabla^{l}, h^{\prime}\left(\rho_{s}\right)\right] \nabla \varrho+\left[\nabla^{l}, \nabla h^{\prime}\left(\rho_{s}\right)\right] \varrho\right) \\
\lesssim\left\|\nabla^{l} u\right\|_{L^{2}}\left\|\left[\nabla^{l}, h^{\prime}\left(\rho_{s}\right)\right] \nabla \varrho\right\|_{L^{2}}+\left\|\nabla^{l} u\right\|_{L^{2}}\left\|\left[\nabla^{l}, \nabla h^{\prime}\left(\rho_{s}\right)\right] \varrho\right\|_{L^{2}} \\
\lesssim\left\|\nabla^{l} u\right\|_{L^{2}}\left(\left\|\nabla h^{\prime}\left(\rho_{s}\right)\right\|_{L^{\infty}}\left\|\nabla^{l} \varrho\right\|_{L^{2}}+\left\|\nabla^{l} h^{\prime}\left(\rho_{s}\right)\right\|_{L^{6}}\|\nabla \varrho\|_{L^{3}}\right) \\
\quad+\left\|\nabla^{l} u\right\|_{L^{2}}\left(\left\|\nabla^{2} h^{\prime}\left(\rho_{s}\right)\right\|_{L^{3}}\left\|\nabla^{l-1} \varrho\right\|_{L^{6}}+\left\|\nabla^{l+1} h^{\prime}\left(\rho_{s}\right)\right\|_{L^{2}}\|\varrho\|_{L^{\infty}}\right) \\
\quad \lesssim\left\|\nabla^{l} u\right\|_{L^{2}}\left(\left\|\nabla^{l} \varrho\right\|_{L^{2}}+\|\nabla \varrho\|_{H^{1}}\right) \lesssim\left\|\nabla^{l} u\right\|_{L^{2}}\left(\left\|\nabla^{l} \varrho\right\|_{L^{2}}+\|\nabla \varrho\|_{L^{2}}\right) .
\end{aligned}
$$

Hence, we may bound the third and fourth terms by

We may thus conclude that

$$
\left\|\nabla^{l} u\right\|_{L^{2}}\left(\left\|\nabla^{l} \varrho\right\|_{L^{2}}+\|\nabla \varrho\|_{L^{2}}\right)
$$

$$
\begin{aligned}
& \int\left(h^{\prime}\left(\rho_{s}\right) \nabla^{l} \varrho \nabla^{l} \operatorname{div}\left(\rho_{s} u\right)+\rho_{s} \nabla^{l} u \cdot \nabla^{l} \nabla\left(h^{\prime}\left(\rho_{s}\right) \varrho\right)\right) \\
& \gtrsim-\left(\left\|\nabla^{l} \varrho\right\|_{L^{2}}+\|\nabla \varrho\|_{L^{2}}\right)\left(\left\|\nabla^{l} u\right\|_{L^{2}}+\|\nabla u\|_{L^{2}}\right) .
\end{aligned}
$$

Next, we compute the following term

$$
\begin{aligned}
& -\int \rho_{s} \nabla^{l} u \cdot \nabla^{l}\left(\frac{1}{\rho_{s}} \nabla \operatorname{div} u\right)=-\int \rho_{s} \nabla^{l} u \cdot \nabla^{l}\left(\nabla\left(\frac{1}{\rho_{s}} \operatorname{div} u\right)-\nabla\left(\frac{1}{\rho_{s}}\right) \operatorname{div} u\right) \\
& =\int\left(\operatorname{div}\left(\rho_{s} \nabla^{l} u\right) \nabla^{l}\left(\frac{1}{\rho_{s}} \operatorname{div} u\right)+\rho_{s} \nabla^{l} u \cdot \nabla^{l}\left(\nabla\left(\frac{1}{\rho_{s}}\right) \operatorname{div} u\right)\right) \\
& =\int\left|\nabla^{l} \operatorname{div} u\right|^{2}+\int \rho_{s} \nabla^{l} \operatorname{div} u\left[\nabla^{l}, \frac{1}{\rho_{s}}\right] \operatorname{div} u
\end{aligned}
$$




$$
+\int \nabla \rho_{s} \cdot \nabla^{l} u \nabla^{l}\left(\frac{1}{\rho_{s}} \operatorname{div} u\right)+\int \rho_{s} \nabla^{l} u \cdot \nabla^{l}\left(\nabla\left(\frac{1}{\rho_{s}}\right) \operatorname{div} u\right) .
$$

For the second term on the right-hand side of (3.23), we employ the commutator estimates (A.3) to obtain: for $l=1$,

$$
\begin{gathered}
\int \rho_{s} \nabla \operatorname{div} u\left[\nabla, \frac{1}{\rho_{s}}\right] \operatorname{div} u \lesssim\|\nabla \operatorname{div} u\|_{L^{2}}\left\|\left[\nabla, \frac{1}{\rho_{s}}\right] \operatorname{div} u\right\|_{L^{2}} \\
\lesssim\left\|\nabla^{2} u\right\|_{L^{2}}\left\|\nabla\left(\frac{1}{\rho_{s}}\right)\right\|_{L^{\infty}}\|\operatorname{div} u\|_{L^{2}} \lesssim\left\|\nabla^{2} u\right\|_{L^{2}}\|\nabla u\|_{L^{2}}
\end{gathered}
$$

for $l \geqslant 2$,

$$
\begin{aligned}
\int & \rho_{s} \nabla^{l} \operatorname{div} u\left[\nabla^{l}, \frac{1}{\rho_{s}}\right] \operatorname{div} u \lesssim\left\|\nabla^{l} \operatorname{div} u\right\|_{L^{2}}\left\|\left[\nabla^{l}, \frac{1}{\rho_{s}}\right] \operatorname{div} u\right\|_{L^{2}} \\
& \lesssim\left\|\nabla^{l+1} u\right\|_{L^{2}}\left(\left\|\nabla\left(\frac{1}{\rho_{s}}\right)\right\|_{L^{\infty}}\left\|\nabla^{l-1} \operatorname{div} u\right\|_{L^{2}}+\left\|\nabla^{l}\left(\frac{1}{\rho_{s}}\right)\right\|_{L^{6}}\|\operatorname{div} u\|_{L^{3}}\right) \\
& \lesssim\left\|\nabla^{l+1} u\right\|_{L^{2}}\left(\left\|\nabla^{l} u\right\|_{L^{2}}+\|\nabla u\|_{H^{1}}\right) \lesssim\left\|\nabla^{l+1} u\right\|_{L^{2}}\left(\left\|\nabla^{l} u\right\|_{L^{2}}+\|\nabla u\|_{L^{2}}\right) .
\end{aligned}
$$

For the third term, we employ the product estimates (A.4) to obtain: for $l=1$,

$$
\begin{aligned}
\int & \nabla \rho_{s} \cdot \nabla u \nabla\left(\frac{1}{\rho_{s}} \operatorname{div} u\right) \lesssim\|\nabla u\|_{L^{2}}\left\|\nabla\left(\frac{1}{\rho_{s}} \operatorname{div} u\right)\right\|_{L^{2}} \\
& \lesssim\|\nabla u\|_{L^{2}}\left(\left\|\frac{1}{\rho_{s}}\right\|_{L^{\infty}}\|\nabla \operatorname{div} u\|_{L^{2}}+\left\|\nabla\left(\frac{1}{\rho_{s}}\right)\right\|_{L^{3}}\|\operatorname{div} u\|_{L^{6}}\right) \\
& \lesssim\left\|\nabla^{2} u\right\|_{L^{2}}\|\nabla u\|_{L^{2}} ;
\end{aligned}
$$

for $l \geqslant 2$,

$$
\begin{aligned}
\int & \nabla \rho_{s} \cdot \nabla^{l} u \cdot \nabla^{l}\left(\frac{1}{\rho_{s}} \operatorname{div} u\right) \lesssim\left\|\nabla^{l} u\right\|_{L^{2}}\left\|\nabla^{l}\left(\frac{1}{\rho_{s}} \operatorname{div} u\right)\right\|_{L^{2}} \\
& \lesssim\left\|\nabla^{l} u\right\|_{L^{2}}\left(\left\|\frac{1}{\rho_{s}}\right\|_{L^{\infty}}\left\|\nabla^{l} \operatorname{div} u\right\|_{L^{2}}+\left\|\nabla^{l}\left(\frac{1}{\rho_{s}}\right)\right\|_{L^{6}}\|\operatorname{div} u\|_{L^{3}}\right) \\
& \lesssim\left\|\nabla^{l} u\right\|_{L^{2}}\left(\left\|\nabla^{l+1} u\right\|_{L^{2}}+\|\nabla u\|_{L^{2}}\right) .
\end{aligned}
$$

Similarly, for the fourth term, we have: for $l=1$,

$$
\begin{aligned}
& \int \rho_{s} \nabla u \cdot \nabla\left(\nabla\left(\frac{1}{\rho_{s}}\right) \operatorname{div} u\right) \lesssim\|\nabla u\|_{L^{2}}\left\|\nabla\left(\nabla\left(\frac{1}{\rho_{s}}\right) \operatorname{div} u\right)\right\|_{L^{2}} \\
& \quad \lesssim\|\nabla u\|_{L^{2}}\left(\left\|\nabla\left(\frac{1}{\rho_{s}}\right)\right\|_{L^{\infty}}\|\nabla \operatorname{div} u\|_{L^{2}}+\left\|\nabla^{2}\left(\frac{1}{\rho_{s}}\right)\right\|_{L^{3}}\|\operatorname{div} u\|_{L^{6}}\right) \\
& \quad \lesssim\left\|\nabla^{2} u\right\|_{L^{2}}\|\nabla u\|_{L^{2}} ;
\end{aligned}
$$

for $l \geqslant 2$,

$$
\begin{gathered}
\int \rho_{s} \nabla^{l} u \cdot \nabla^{l}\left(\nabla\left(\frac{1}{\rho_{s}}\right) \operatorname{div} u\right) \lesssim\left\|\nabla^{l} u\right\|_{L^{2}}\left\|\nabla^{l}\left(\nabla\left(\frac{1}{\rho_{s}}\right) \operatorname{div} u\right)\right\|_{L^{2}} \\
\lesssim\left\|\nabla^{l} u\right\|_{L^{2}}\left(\left\|\nabla\left(\frac{1}{\rho_{s}}\right)\right\|_{L^{\infty}}\left\|\nabla^{l} \operatorname{div} u\right\|_{L^{2}}+\left\|\nabla^{l+1}\left(\frac{1}{\rho_{s}}\right)\right\|_{L^{2}}\|\operatorname{div} u\|_{L^{\infty}}\right) \\
\lesssim\left\|\nabla^{l} u\right\|_{L^{2}}\left(\left\|\nabla^{l+1} u\right\|_{L^{2}}+\|\nabla u\|_{H^{2}}\right) \lesssim\left\|\nabla^{l} u\right\|_{L^{2}}\left(\left\|\nabla^{l+1} u\right\|_{L^{2}}+\|\nabla u\|_{L^{2}}\right) .
\end{gathered}
$$

In light of (3.24) - (3.29) and Cauchy's inequality, we deduce that the last three terms in (3.23) are bounded by that for any small constant $\eta>0$,

$$
\eta\left\|\nabla^{l+1} u\right\|_{L^{2}}^{2}+C_{\eta}\left(\left\|\nabla^{l} u\right\|_{L^{2}}^{2}+\|\nabla u\|_{L^{2}}^{2}\right)
$$


We now compute the term

$$
\begin{aligned}
- & \int \rho_{s} \nabla^{l} u \cdot \nabla^{l}\left(\frac{1}{\rho_{s}} \Delta u\right)=-\int \rho_{s} \nabla^{l} u \cdot \nabla^{l}\left(\operatorname{div}\left(\frac{1}{\rho_{s}} \nabla u\right)-\nabla\left(\frac{1}{\rho_{s}}\right) \cdot \nabla u\right) \\
= & \int \nabla\left(\rho_{s} \nabla^{l} u\right) \cdot \nabla^{l}\left(\frac{1}{\rho_{s}} \nabla u\right)+\rho_{s} \nabla^{l} u \cdot \nabla^{l}\left(\nabla\left(\frac{1}{\rho_{s}}\right) \cdot \nabla u\right) \\
= & \int\left|\nabla^{l+1} u\right|^{2}+\int \rho_{s} \nabla^{l+1} u \cdot\left[\nabla^{l}, \frac{1}{\rho_{s}}\right] \nabla u \\
& +\int \nabla \rho_{s} \cdot \nabla^{l} u \cdot \nabla^{l}\left(\frac{1}{\rho_{s}} \nabla u\right)+\int \rho_{s} \nabla^{l} u \cdot \nabla^{l}\left(\nabla\left(\frac{1}{\rho_{s}}\right) \cdot \nabla u\right) .
\end{aligned}
$$

So as for (3.23), the last three terms in (3.30) are also bounded by

$$
\eta\left\|\nabla^{l+1} u\right\|_{L^{2}}^{2}+C_{\eta}\left(\left\|\nabla^{l} u\right\|_{L^{2}}^{2}+\|\nabla u\|_{L^{2}}^{2}\right) \text {. }
$$

Hence, we may conclude that

$$
\begin{gathered}
-\int \rho_{s} \nabla^{l} u \cdot \nabla^{l}\left(\frac{1}{\rho_{s}}\left(\mu \Delta u+\left(\mu+\mu^{\prime}\right) \nabla \operatorname{div} u\right)\right) \\
\gtrsim\left\|\nabla^{l+1} u\right\|_{L^{2}}^{2}-C\left(\left\|\nabla^{l} u\right\|_{L^{2}}^{2}+\|\nabla u\|_{L^{2}}^{2}\right) .
\end{gathered}
$$

We may simply bound the Poisson term as

$$
-\int \rho_{s} \nabla^{l} u \cdot \nabla^{l} \nabla \Phi \lesssim\left\|\nabla^{l} u\right\|_{L^{2}}\left\|\nabla^{l} \nabla \Phi\right\|_{L^{2}} \lesssim\left\|\nabla^{l} u\right\|_{L^{2}}\left(\|\varrho\|_{L^{2}}+\left\|\nabla^{l-1} \varrho\right\|_{L^{2}}\right) .
$$

Then, we obtain

$$
-\int \rho_{s} \nabla^{l} u \cdot \nabla^{l} \nabla \Phi \gtrsim-\left\|\nabla^{l} u\right\|_{L^{2}}\left(\|\varrho\|_{L^{2}}+\left\|\nabla^{l-1} \varrho\right\|_{L^{2}}\right) .
$$

Now, we estimate the nonlinear terms on the right-hand side of (3.14). By the commutator notation (A.2), we have

$$
\begin{aligned}
& -\int h^{\prime}\left(\rho_{s}\right) \nabla^{l} \varrho \nabla^{l} \operatorname{div}(\varrho u)=-\int h^{\prime}\left(\rho_{s}\right) \nabla^{l} \varrho \nabla^{l}(u \cdot \nabla \varrho+\varrho \operatorname{div} u) \\
& =-\int h^{\prime}\left(\rho_{s}\right) u \cdot \nabla \nabla^{l} \varrho \nabla^{l} \varrho-\int h^{\prime}\left(\rho_{s}\right) \nabla^{l} \varrho\left[\nabla^{l}, u\right] \cdot \nabla \varrho-\int h^{\prime}\left(\rho_{s}\right) \nabla^{l} \varrho \nabla^{l}(\varrho \operatorname{div} u) .
\end{aligned}
$$

By the integration by parts, we obtain

$$
\begin{aligned}
& -\int h^{\prime}\left(\rho_{s}\right) u \cdot \nabla \nabla^{l} \varrho \nabla^{l} \varrho=-\frac{1}{2} \int h^{\prime}\left(\rho_{s}\right) u \cdot \nabla\left|\nabla^{l} \varrho\right|^{2}=\frac{1}{2} \int \operatorname{div}\left(h^{\prime}\left(\rho_{s}\right) u\right)\left|\nabla^{l} \varrho\right|^{2} \\
& \quad \lesssim\left(\|u\|_{L^{\infty}}+\|\operatorname{div} u\|_{L^{\infty}}\right)\left\|\nabla^{l} \varrho\right\|_{L^{2}}^{2} \lesssim \delta\left\|\nabla^{l} \varrho\right\|_{L^{2}}^{2} .
\end{aligned}
$$

By the commutator estimates (A.3), we obtain

$$
\begin{aligned}
& -\int h^{\prime}\left(\rho_{s}\right) \nabla^{l} \varrho\left[\nabla^{l}, u\right] \cdot \nabla \varrho \lesssim\left\|\nabla^{l} \varrho\right\|_{L^{2}}\left\|\left[\nabla^{l}, u\right] \cdot \nabla \varrho\right\|_{L^{2}} \\
& \quad \lesssim\left\|\nabla^{l} \varrho\right\|_{L^{2}}\left(\|\nabla u\|_{L^{\infty}}\left\|\nabla^{l} \varrho\right\|_{L^{2}}+\left\|\nabla^{l} u\right\|_{L^{6}}\|\nabla \varrho\|_{L^{3}}\right) \\
& \quad \lesssim \delta\left(\left\|\nabla^{l} \varrho\right\|_{L^{2}}^{2}+\left\|\nabla^{l+1} u\right\|_{L^{2}}^{2}\right) .
\end{aligned}
$$

By the product estimates (A.4), we obtain

$$
\begin{aligned}
& -\int h^{\prime}\left(\rho_{s}\right) \nabla^{l} \varrho \nabla^{l}(\varrho \operatorname{div} u) \lesssim\left\|\nabla^{l} \varrho\right\|_{L^{2}}\left\|\nabla^{l}(\varrho \operatorname{div} u)\right\|_{L^{2}} \\
& \quad \lesssim\left\|\nabla^{l} \varrho\right\|_{L^{2}}\left(\|\varrho\|_{L^{\infty}}\left\|\nabla^{l} \operatorname{div} u\right\|_{L^{2}}+\left\|\nabla^{l} \varrho\right\|_{L^{2}}\|\operatorname{div} u\|_{L^{\infty}}\right)
\end{aligned}
$$




$$
\lesssim \delta\left(\left\|\nabla^{l} \varrho\right\|_{L^{2}}^{2}+\left\|\nabla^{l+1} u\right\|_{L^{2}}^{2}\right)
$$

Hence, we deduce from (3.34)-(3.37) that

$$
-\int h^{\prime}\left(\rho_{s}\right) \nabla^{l} \varrho \nabla^{l} \operatorname{div}(\varrho u) \lesssim \delta\left(\left\|\nabla^{l} \varrho\right\|_{L^{2}}^{2}+\left\|\nabla^{l+1} u\right\|_{L^{2}}^{2}\right) .
$$

We use the product estimates (A.4) to obtain

$$
\begin{aligned}
& -\int \rho_{s} \nabla^{l} u \cdot \nabla^{l}(u \cdot \nabla u) \lesssim\left\|\nabla^{l} u\right\|_{L^{2}}\left\|\nabla^{l}(u \cdot \nabla u)\right\|_{L^{2}} \\
& \quad \lesssim\left\|\nabla^{l} u\right\|_{L^{2}}\left(\|u\|_{L^{\infty}}\left\|\nabla^{l} \nabla u\right\|_{L^{2}}+\left\|\nabla^{l} u\right\|_{L^{2}}\|\nabla u\|_{L^{\infty}}\right) \\
& \quad \lesssim \delta\left(\left\|\nabla^{l} u\right\|_{L^{2}}^{2}+\left\|\nabla^{l+1} u\right\|_{L^{2}}^{2}\right) .
\end{aligned}
$$

By the integration by parts, we deduce

$$
\begin{aligned}
& -\int \rho_{s} \nabla^{l} u \cdot \nabla^{l} \nabla \mathcal{R}=\int \nabla \rho_{s} \cdot \nabla^{l} u \nabla^{l} \mathcal{R}+\int \rho_{s} \nabla^{l} \operatorname{div} u \nabla^{l} \mathcal{R} \\
& \quad \lesssim\left\|\nabla \rho_{s}\right\|_{L^{3}}\left\|\nabla^{l} u\right\|_{L^{6}}\left\|\nabla^{l} \mathcal{R}\right\|_{L^{2}}+\left\|\nabla^{l+1} u\right\|_{L^{2}}\left\|\nabla^{l} \mathcal{R}\right\|_{L^{2}} \\
& \quad \lesssim \delta\left(\|\nabla \varrho\|_{L^{2}}^{2}+\left\|\nabla^{l} \varrho\right\|_{L^{2}}^{2}+\left\|\nabla^{l+1} u\right\|_{L^{2}}^{2}\right) .
\end{aligned}
$$

Here we have used the nonlinear estimates A.5 of $\mathcal{R}$ stated in Lemma A.3.

For the last term, we integrate by parts to have

$$
\begin{aligned}
& \int \rho_{s} \nabla^{l} u \cdot \nabla^{l}\left(\left(\frac{1}{\varrho+\rho_{s}}-\frac{1}{\rho_{s}}\right) \nabla^{2} u\right) \\
& =-\int \nabla \rho_{s} \nabla^{l} u \cdot \nabla^{l-1}\left(\left(\frac{1}{\varrho+\rho_{s}}-\frac{1}{\rho_{s}}\right) \nabla^{2} u\right)-\int \rho_{s} \nabla^{l+1} u \cdot \nabla^{l-1}\left(\left(\frac{1}{\varrho+\rho_{s}}-\frac{1}{\rho_{s}}\right) \nabla^{2} u\right) \\
& \lesssim\left\|\nabla^{l} u\right\|_{L^{6}}\left\|\nabla^{l-1}\left(\left(\frac{1}{\varrho+\rho_{s}}-\frac{1}{\rho_{s}}\right) \nabla^{2} u\right)\right\|_{L^{6 / 5}} \\
& +\left\|\nabla^{l+1} u\right\|_{L^{2}}\left\|\nabla^{l-1}\left(\left(\frac{1}{\varrho+\rho_{s}}-\frac{1}{\rho_{s}}\right) \nabla^{2} u\right)\right\|_{L^{2}} \\
& \lesssim\left\|\nabla^{l+1} u\right\|_{L^{2}}\left(\left\|\frac{1}{\varrho+\rho_{s}}-\frac{1}{\rho_{s}}\right\|_{L^{3}}\left\|\nabla^{l+1} u\right\|_{L^{2}}+\left\|\nabla^{l-1}\left(\frac{1}{\varrho+\rho_{s}}-\frac{1}{\rho_{s}}\right)\right\|_{L^{2}}\left\|\nabla^{2} u\right\|_{L^{3}}\right) \\
& +\left\|\nabla^{l+1} u\right\|_{L^{2}}\left(\left\|\frac{1}{\varrho+\rho_{s}}-\frac{1}{\rho_{s}}\right\|_{L^{\infty}}\left\|\nabla^{l+1} u\right\|_{L^{2}}+\left\|\nabla^{l-1}\left(\frac{1}{\varrho+\rho_{s}}-\frac{1}{\rho_{s}}\right)\right\|_{L^{6}}\left\|\nabla^{2} u\right\|_{L^{3}}\right) \\
& \lesssim \delta\left(\left\|\nabla^{l+1} u\right\|_{L^{2}}^{2}+\left\|\nabla^{l-1} \varrho\right\|_{L^{2}}^{2}+\left\|\nabla^{l} \varrho\right\|_{L^{2}}^{2}\right) \text {. }
\end{aligned}
$$

Hence, we deduce that

$$
\begin{aligned}
& \int \rho_{s} \nabla^{l} u \cdot \nabla^{l}\left(\left(\frac{1}{\varrho+\rho_{s}}-\frac{1}{\rho_{s}}\right)\left(\mu \Delta u+\left(\mu+\mu^{\prime}\right) \nabla \operatorname{div} u\right)\right) \\
& \quad \lesssim \delta\left(\left\|\nabla^{l+1} u\right\|_{L^{2}}^{2}+\left\|\nabla^{l-1} \varrho\right\|_{L^{2}}^{2}+\left\|\nabla^{l} \varrho\right\|_{L^{2}}^{2}\right) .
\end{aligned}
$$

Plugging the estimates (3.22), (3.31)-(3.33) and (3.38)-(3.42) into (3.14), we then obtain

$$
\begin{aligned}
& \frac{d}{d t}\left(\int h^{\prime}\left(\rho_{s}\right)\left|\nabla^{l} \varrho\right|^{2}+\rho_{s}\left|\nabla^{l} u\right|^{2}\right)+\left\|\nabla^{l+1} u\right\|_{L^{2}}^{2} \\
& \quad \lesssim \delta\left(\left\|\nabla^{l+1} u\right\|_{L^{2}}^{2}+\left\|\nabla^{l} u\right\|_{L^{2}}^{2}+\left\|\nabla^{l} \varrho\right\|_{L^{2}}^{2}+\left\|\nabla^{l-1} \varrho\right\|_{L^{2}}^{2}+\|\nabla \varrho\|_{L^{2}}^{2}+\|\varrho\|_{L^{2}}^{2}\right)
\end{aligned}
$$




$$
+\left\|\nabla^{l} u\right\|_{L^{2}}^{2}+\|\nabla u\|_{L^{2}}^{2}+\left(\left\|\nabla^{l} u\right\|_{L^{2}}+\|\nabla u\|_{L^{2}}\right)\left(\left\|\nabla^{l} \varrho\right\|_{L^{2}}+\|\varrho\|_{L^{2}}\right) .
$$

By the interpolation and Young's inequality, since $\delta$ is small, we deduce (3.13).

Now we recover the dissipation for $\varrho$, and we first deal with $\varrho$ itself.

Lemma 3.3. It holds that

$$
\frac{d}{d t} \int u \cdot \nabla\left(h^{\prime}\left(\rho_{s}\right) \varrho\right)+\|\varrho\|_{L^{2}}^{2}+\|\nabla \varrho\|_{L^{2}}^{2} \lesssim\left\|\nabla^{2} u\right\|_{L^{2}}^{2}+\|\nabla u\|_{L^{2}}^{2} .
$$

Proof. Multiplying the second equation in (3.5) by $\nabla\left(h^{\prime}\left(\rho_{s}\right) \varrho\right)$ and then integrating over $\mathbb{R}^{3}$, by Cauchy's inequality and the nonlinear estimates (A.5), we obtain

$$
\begin{aligned}
& \int \partial_{t} u \cdot \nabla\left(h^{\prime}\left(\rho_{s}\right) \varrho\right)+\left\|\nabla\left(h^{\prime}\left(\rho_{s}\right) \varrho\right)\right\|_{L^{2}}^{2}-\int \nabla \Phi \cdot \nabla\left(h^{\prime}\left(\rho_{s}\right) \varrho\right) \\
& \quad \lesssim\left(\left\|\nabla^{2} u\right\|_{L^{2}}+\|u \cdot \nabla u\|_{L^{2}}+\|\nabla \mathcal{R}\|_{L^{2}}+\left\|\left(\frac{1}{\varrho+\rho_{s}}-\frac{1}{\rho_{s}}\right) \nabla^{2} u\right\|_{L^{2}}\right)\left\|\nabla\left(h^{\prime}\left(\rho_{s}\right) \varrho\right)\right\|_{L^{2}} \\
& \quad \lesssim\left\|\nabla^{2} u\right\|_{L^{2}}^{2}+\delta\|\nabla \varrho\|_{L^{2}}^{2} .
\end{aligned}
$$

By the first equation in (3.5), we integrate by parts for both $t$ - and $x$-variables to have

$$
\begin{aligned}
& -\int \partial_{t} u \cdot \nabla\left(h^{\prime}\left(\rho_{s}\right) \varrho\right)=-\frac{d}{d t} \int u \cdot \nabla\left(h^{\prime}\left(\rho_{s}\right) \varrho\right)+\int u \cdot \nabla\left(h^{\prime}\left(\rho_{s}\right) \partial_{t} \varrho\right) \\
& =-\frac{d}{d t} \int u \cdot \nabla\left(h^{\prime}\left(\rho_{s}\right) \varrho\right)+\int h^{\prime}\left(\rho_{s}\right) \operatorname{div} u\left(u \cdot \nabla \varrho+\varrho \operatorname{div} u+\rho_{s} \operatorname{div} u+u \cdot \nabla \rho_{s}\right) \\
& \quad \leqslant-\frac{d}{d t} \int u \cdot \nabla\left(h^{\prime}\left(\rho_{s}\right) \varrho\right)+C\|\nabla u\|_{L^{2}}^{2} .
\end{aligned}
$$

By the integration by parts and the Poisson equation in (3.5), we obtain

$$
-\int \nabla \Phi \cdot \nabla\left(h^{\prime}\left(\rho_{s}\right) \varrho\right)=\int \Delta \Phi h^{\prime}\left(\rho_{s}\right) \varrho=\int h^{\prime}\left(\rho_{s}\right) \varrho^{2} .
$$

Plugging (3.46)-(3.47) into (3.45), since $\delta$ is small, we deduce (3.44) by noticing that

$$
\|\varrho\|_{L^{2}}^{2}+\|\nabla \varrho\|_{L^{2}}^{2} \lesssim \int h^{\prime}\left(\rho_{s}\right) \varrho^{2}+\left\|\nabla\left(h^{\prime}\left(\rho_{s}\right) \varrho\right)\right\|_{L^{2}}^{2} .
$$

We thus conclude the lemma.

We next derive the dissipation estimates for the derivatives of $\varrho$.

Lemma 3.4. For $l=1, \ldots, k-1$, we have

$$
\frac{d}{d t} \int \nabla^{l} u \cdot \nabla \nabla^{l} \varrho+\left\|\nabla^{l} \varrho\right\|_{L^{2}}^{2}+\left\|\nabla^{l+1} \varrho\right\|_{L^{2}}^{2} \lesssim\left\|\nabla^{l+2} u\right\|_{L^{2}}^{2}+\|\nabla u\|_{L^{2}}^{2}+\|\varrho\|_{L^{2}}^{2} .
$$

Proof. Applying $\nabla^{l}$ to the second equation in (3.5), multiplying the resulting identity by $\nabla^{l} \nabla \varrho$ and then integrating over $\mathbb{R}^{3}$, we obtain

$$
\begin{aligned}
\int \nabla^{l} \partial_{t} u \cdot \nabla^{l} \nabla \varrho+\int h^{\prime}\left(\rho_{s}\right)\left|\nabla^{l+1} \varrho\right|^{2}-\int \nabla^{l} \nabla \Phi \cdot \nabla^{l} \nabla \varrho \\
\lesssim\left(\left\|\left[\nabla^{l+1}, h^{\prime}\left(\rho_{s}\right)\right] \varrho\right\|_{L^{2}}+\left\|\nabla^{l+2} u\right\|_{L^{2}}+\left\|\nabla^{l}(u \cdot \nabla u)\right\|_{L^{2}}\right. \\
\left.\quad+\left\|\nabla^{l+1} \mathcal{R}\right\|_{L^{2}}+\left\|\nabla^{l}\left(\left(\frac{1}{\varrho+\rho_{s}}-\frac{1}{\rho_{s}}\right) \nabla^{2} u\right)\right\|_{L^{2}}\right)\left\|\nabla^{l+1} \varrho\right\|_{L^{2}}
\end{aligned}
$$

As (3.46), we deduce

$$
\begin{aligned}
& -\int \nabla^{l} \partial_{t} u \cdot \nabla \nabla^{l} \varrho=-\frac{d}{d t} \int \nabla^{l} u \cdot \nabla \nabla^{l} \varrho+\int \nabla^{l} u \cdot \nabla \nabla^{l} \partial_{t} \varrho \\
& =-\frac{d}{d t} \int \nabla^{l} u \cdot \nabla \nabla^{l} \varrho+\int \nabla^{l} \operatorname{div} u \nabla^{l}\left(u \cdot \nabla \varrho+\varrho \operatorname{div} u+\rho_{s} \operatorname{div} u+u \cdot \nabla \rho_{s}\right)
\end{aligned}
$$




$$
\begin{aligned}
\leqslant & -\frac{d}{d t} \int \nabla^{l} u \cdot \nabla \nabla^{l} \varrho+\left\|\nabla^{l+1} u\right\|_{L^{2}}^{2}+\left\|\nabla^{l}(u \cdot \nabla \varrho)\right\|_{L^{2}}^{2} \\
& +\left\|\nabla^{l}(\varrho \operatorname{div} u)\right\|_{L^{2}}^{2}+\left\|\nabla^{l}\left(\rho_{s} \operatorname{div} u\right)\right\|_{L^{2}}^{2}+\left\|\nabla^{l}\left(u \cdot \nabla \rho_{s}\right)\right\|_{L^{2}}^{2} .
\end{aligned}
$$

As (3.47), we have

$$
-\int \nabla^{l} \nabla \Phi \cdot \nabla^{l} \nabla \varrho=\int \nabla^{l} \Delta \Phi \nabla^{l} \varrho=\left\|\nabla^{l} \varrho\right\|_{L^{2}}^{2} .
$$

Plugging (3.51)-(3.52) into (3.50), and applying the product estimates (A.4), Lemma A.1 and the nonlinear estimates (A.5) as in Lemma 3.2, we obtain

$$
\begin{aligned}
& \frac{d}{d t} \int \nabla^{l} u \cdot \nabla \nabla^{l} \varrho+\int h^{\prime}\left(\rho_{s}\right)\left|\nabla^{l+1} \varrho\right|^{2}+\left\|\nabla^{l} \varrho\right\|_{L^{2}}^{2} \\
& \quad \lesssim\left\|\left[\nabla^{l+1}, h^{\prime}\left(\rho_{s}\right)\right] \varrho\right\|_{L^{2}}\left\|\nabla^{l+1} \varrho\right\|_{L^{2}} \\
& \quad+\left(\left\|\nabla^{l+2} u\right\|_{L^{2}}+\|\nabla u\|_{L^{2}}+\delta\left(\|\nabla \varrho\|_{L^{2}}+\left\|\nabla^{l+1} \varrho\right\|_{L^{2}}\right)\right)\left\|\nabla^{l+1} \varrho\right\|_{L^{2}} .
\end{aligned}
$$

By the commutator estimates (A.3), we obtain

$$
\begin{aligned}
\left\|\left[\nabla^{l+1}, h^{\prime}\left(\rho_{s}\right)\right] \varrho\right\|_{L^{2}} & \lesssim\left\|\nabla h^{\prime}\left(\rho_{s}\right)\right\|_{L^{\infty}}\left\|\nabla^{l} \varrho\right\|_{L^{2}}+\left\|\nabla^{l+1} h^{\prime}\left(\rho_{s}\right)\right\|_{L^{3}}\|\varrho\|_{L^{6}} \\
& \lesssim\left\|\nabla^{l} \varrho\right\|_{L^{2}}+\|\nabla \varrho\|_{L^{2}} .
\end{aligned}
$$

Plugging (3.54) into (3.53), by Cauchy's inequality and since $\delta$ is small, we have

$$
\begin{aligned}
& \frac{d}{d t} \int \nabla^{l} u \cdot \nabla \nabla^{l} \varrho+\left\|\nabla^{l+1} \varrho\right\|_{L^{2}}^{2}+\left\|\nabla^{l} \varrho\right\|_{L^{2}}^{2} \\
& \quad \lesssim\left\|\nabla^{l+2} u\right\|_{L^{2}}^{2}+\|\nabla u\|_{L^{2}}^{2}+\left\|\nabla^{l} \varrho\right\|_{L^{2}}^{2}+\|\nabla \varrho\|_{L^{2}}^{2} .
\end{aligned}
$$

By the interpolation and Young's inequality, we further deduce (3.49) from (3.55).

3.2. Proof of Theorem 1.1. In this subsection, we will prove Theorem 1.1. Multiplying the estimates (3.49) of Lemma 3.4 with $l=k-1$ by a small fixed constant $\epsilon_{1}$, and then adding it to the estimates (3.44) of Lemma 3.3, by the interpolation and Young's inequality, we deduce

$$
\frac{d}{d t}\left(\int u \cdot \nabla\left(h^{\prime}\left(\rho_{s}\right) \varrho\right)+\epsilon_{1} \int \nabla^{k-1} u \cdot \nabla^{k} \varrho\right)+\|\varrho\|_{H^{k}}^{2} \lesssim\left\|\nabla^{k+1} u\right\|_{L^{2}}^{2}+\|\nabla u\|_{L^{2}}^{2} .
$$

Multiplying (3.56) by a small fixed constant $\epsilon_{2}$ and then adding it to the estimates (3.13) of Lemma 3.2 with $l=k$, we obtain

$$
\begin{aligned}
& \frac{d}{d t}\left(\int h^{\prime}\left(\rho_{s}\right)\left|\nabla^{k} \varrho\right|^{2}+\rho_{s}\left|\nabla^{k} u\right|^{2}+\epsilon_{2}\left(\int u \cdot \nabla\left(h^{\prime}\left(\rho_{s}\right) \varrho\right)+\epsilon_{1} \int \nabla^{k-1} u \cdot \nabla^{k} \varrho\right)\right) \\
& \quad+\left\|\nabla^{k+1} u\right\|_{L^{2}}^{2}+\|\varrho\|_{H^{k}}^{2} \lesssim(\varepsilon+\delta)\|\varrho\|_{H^{k}}^{2}+C_{\varepsilon}\left(\left\|\nabla^{k} u\right\|_{L^{2}}^{2}+\|\nabla u\|_{L^{2}}^{2}\right) .
\end{aligned}
$$

Taking $\varepsilon$ sufficiently small in (3.57) and since $\delta$ is small, by the interpolation and Young's inequality, we then have

$$
\begin{aligned}
& \frac{d}{d t}\left(\int h^{\prime}\left(\rho_{s}\right)\left|\nabla^{k} \varrho\right|^{2}+\rho_{s}\left|\nabla^{k} u\right|^{2}+\epsilon_{2}\left(\int u \cdot \nabla\left(h^{\prime}\left(\rho_{s}\right) \varrho\right)+\epsilon_{1} \int \nabla^{k-1} u \cdot \nabla^{k} \varrho\right)\right) \\
& \quad+\|\nabla u\|_{H^{k}}^{2}+\|\varrho\|_{H^{k}}^{2} \lesssim\|\nabla u\|_{L^{2}}^{2} .
\end{aligned}
$$

Multiplying (3.58) by a small fixed constant $\epsilon_{3}$ and then adding it to the estimates (3.7) of Lemma 3.1, since $\delta$ is small, we deduce

$$
\begin{aligned}
& \frac{d}{d t}\left(\int\left(h^{\prime}\left(\rho_{s}\right) \varrho^{2}+\rho_{s}|u|^{2}+|\nabla \Phi|^{2}\right)+\epsilon_{3}\left(\int h^{\prime}\left(\rho_{s}\right)\left|\nabla^{k} \varrho\right|^{2}+\rho_{s}\left|\nabla^{k} u\right|^{2}\right.\right. \\
& \left.\left.\quad+\epsilon_{2}\left(\int u \cdot \nabla\left(h^{\prime}\left(\rho_{s}\right) \varrho\right)+\epsilon_{1} \int \nabla^{k-1} u \cdot \nabla^{k} \varrho\right)\right)\right)+\|\nabla u\|_{H^{k}}^{2}+\|\varrho\|_{H^{k}}^{2} \leqslant 0 .
\end{aligned}
$$


Note that the expression under the time differentiation in (3.59) with properly small $\epsilon_{1}, \epsilon_{2}$ and $\epsilon_{3}$ is equivalent to $\|\varrho\|_{H^{k}}^{2}+\|u\|_{H^{k}}^{2}+\|\nabla \Phi\|_{L^{2}}^{2}$. Hence, integrating (3.59) directly in time, we obtain (1.8). By a standard continuity argument, we then close the a priori estimates (3.6) if we assume at initial time that $\left\|\varrho_{0}\right\|_{H^{k}}+\left\|u_{0}\right\|_{H^{k}}+\left\|\nabla \Phi_{0}\right\|_{L^{2}}$ is sufficiently small. The global solution then follows by a standard continuity argument combined with the local existence of solutions. The proof of Theorem 1.1 is thus completed.

\section{Time DECAY With SMALl DOPING PROFILE}

In this section, we will derive the time decay rates of the solution to (1.1) towards the steady state. For this, we need to require that the doping profile is of small variation, that is, $b(x)$ is near the constant $\bar{b}$. Then according to Proposition [2.1, $\rho_{s}(x)$ is near $\bar{\rho}$ with $\bar{\rho}=\bar{b}$. Owing to this fact, we may rewrite (3.5) as:

$$
\left\{\begin{array}{l}
\partial_{t} \varrho+\bar{\rho} \operatorname{div} u=-\operatorname{div}\left(\left(\varrho+\rho_{s}-\bar{\rho}\right) u\right) \\
\partial_{t} u+h^{\prime}(\bar{\rho}) \nabla \varrho-\frac{1}{\bar{\rho}}\left(\mu \Delta u+\left(\mu+\mu^{\prime}\right) \nabla \operatorname{div} u\right)-\nabla \Phi \\
\quad=\left(\frac{1}{\varrho+\rho_{s}}-\frac{1}{\bar{\rho}}\right)\left(\mu \Delta u+\left(\mu+\mu^{\prime}\right) \nabla \operatorname{div} u\right)-u \cdot \nabla u-\nabla \mathcal{R}-\nabla\left(\left(h^{\prime}\left(\rho_{s}\right)-h^{\prime}(\bar{\rho})\right) \varrho\right), \\
\Delta \Phi=\varrho \\
\left.(\varrho, u)\right|_{t=0}=\left(\varrho_{0}, u_{0}\right) .
\end{array}\right.
$$

4.1. Energy estimates. In this subsection, under the assumptions of Theorem 1.3, we will derive the further energy estimates for the global solutions to the Navier-Stokes-Poisson equations (4.1) obtained in Theorem 1.1. By Theorem 1.1. the assumptions of Theorem 1.3 and Proposition 2.1, we have that

$$
\|(\varrho, u)(t)\|_{H^{k}}+\|\nabla \Phi(t)\|_{L^{2}}+\left\|\rho_{s}-\bar{\rho}\right\|_{H^{k+1}} \leqslant \delta
$$

for some small constant $\delta>0$.

We first derive the energy estimates for the derivatives of the solution of order $l$ with $l \in[0, k]$. Note that now $l$ is not required to be an integer.

Lemma 4.1. For $0 \leqslant l \leqslant k$, we have

$$
\begin{aligned}
& \frac{d}{d t} \int\left(h^{\prime}(\bar{\rho})\left|\nabla^{l} \varrho\right|^{2}+\bar{\rho}\left|\nabla^{l} u\right|^{2}+\left|\nabla^{l} \nabla \Phi\right|^{2}\right)+\left\|\nabla^{l+1} u\right\|_{L^{2}}^{2} \\
& \quad \lesssim \delta\left(\left\|\nabla^{l} \varrho\right\|_{L^{2}}^{2}+\left\|\nabla^{l} \nabla \Phi\right\|_{L^{2}}^{2}+\left\|\nabla^{l} u\right\|_{L^{2}}^{2}+\|\varrho\|_{L^{\infty}}^{2}+\|u\|_{L^{\infty}}^{2}+\|\nabla u\|_{L^{3}}^{2}+\left\|\nabla^{2} u\right\|_{L^{3}}^{2}\right) .
\end{aligned}
$$

Proof. Applying $\nabla^{l}$ to the first two equations in (4.1) and then multiplying the resulting identities by $h^{\prime}(\bar{\rho}) \nabla^{l} \varrho$ and $\bar{\rho} \nabla^{l} u$ respectively, summing up them and then integrating over $\mathbb{R}^{3}$, we obtain

$$
\begin{aligned}
\frac{1}{2} \frac{d}{d t} & \int\left(h^{\prime}(\bar{\rho})\left|\nabla^{l} \varrho\right|^{2}+\bar{\rho}\left|\nabla^{l} u\right|^{2}\right)+\int \mu\left|\nabla^{l+1} u\right|^{2}+\left(\mu+\mu^{\prime}\right)\left|\nabla^{l} \operatorname{div} u\right|^{2}-\int \bar{\rho} \nabla^{l} u \cdot \nabla^{l} \nabla \Phi \\
= & -\int h^{\prime}(\bar{\rho}) \nabla^{l} \varrho \nabla^{l} \operatorname{div}\left(\left(\varrho+\rho_{s}-\bar{\rho}\right) u\right)-\int \bar{\rho} \nabla^{l} u \cdot \nabla^{l}(u \cdot \nabla u) \\
& -\int \bar{\rho} \nabla^{l} u \cdot \nabla^{l} \nabla \mathcal{R}-\int \bar{\rho} \nabla^{l} u \cdot \nabla^{l} \nabla\left(\left(h^{\prime}\left(\rho_{s}\right)-h^{\prime}(\bar{\rho})\right) \varrho\right) \\
& +\int \bar{\rho} \nabla^{l} u \cdot \nabla^{l}\left(\left(\frac{1}{\varrho+\rho_{s}}-\frac{1}{\bar{\rho}}\right)\left(\mu \Delta u+\left(\mu+\mu^{\prime}\right) \nabla \operatorname{div} u\right)\right) .
\end{aligned}
$$

First, we estimate the terms on the right-hand side of (4.4). By the commutator notation (A.2), we obtain

$$
-\int h^{\prime}(\bar{\rho}) \nabla^{l} \varrho \nabla^{l} \operatorname{div}\left(\left(\varrho+\rho_{s}-\bar{\rho}\right) u\right)
$$




$$
\begin{aligned}
= & -\int h^{\prime}(\bar{\rho}) \nabla^{l} \varrho \nabla^{l}\left(u \cdot \nabla\left(\varrho+\rho_{s}-\bar{\rho}\right)+\left(\varrho+\rho_{s}-\bar{\rho}\right) \operatorname{div} u\right) \\
= & -\int h^{\prime}(\bar{\rho}) u \cdot \nabla \nabla^{l} \varrho \nabla^{l} \varrho-\int h^{\prime}(\bar{\rho}) \nabla^{l} \varrho\left[\nabla^{l}, u\right] \cdot \nabla \varrho \\
& -\int h^{\prime}(\bar{\rho}) \nabla^{l} \varrho \nabla^{l}\left(u \cdot \nabla\left(\rho_{s}-\bar{\rho}\right)\right)-\int h^{\prime}(\bar{\rho}) \nabla^{l} \varrho \nabla^{l}\left(\left(\varrho+\rho_{s}-\bar{\rho}\right) \operatorname{div} u\right) .
\end{aligned}
$$

By the integration by parts, we obtain

$$
\begin{aligned}
& -\int h^{\prime}(\bar{\rho}) u \cdot \nabla \nabla^{l} \varrho \nabla^{l} \varrho=-\frac{1}{2} \int h^{\prime}(\bar{\rho}) u \cdot \nabla\left|\nabla^{l} \varrho\right|^{2}=\frac{1}{2} \int h^{\prime}(\bar{\rho}) \operatorname{div} u\left|\nabla^{l} \varrho\right|^{2} \\
& \quad \lesssim\|\operatorname{div} u\|_{L^{\infty}}\left\|\nabla^{l} \varrho\right\|_{L^{2}}^{2} \lesssim \delta\left\|\nabla^{l} \varrho\right\|_{L^{2}}^{2} .
\end{aligned}
$$

By the commutator estimates (A.3), we obtain

$$
\begin{aligned}
& -\int h^{\prime}(\bar{\rho}) \nabla^{l} \varrho\left[\nabla^{l}, u\right] \cdot \nabla \varrho \lesssim\left\|\nabla^{l} \varrho\right\|_{L^{2}}\left\|\left[\nabla^{l}, u\right] \cdot \nabla \varrho\right\|_{L^{2}} \\
& \quad \lesssim\left\|\nabla^{l} \varrho\right\|_{L^{2}}\left(\|\nabla u\|_{L^{\infty}}\left\|\nabla^{l} \varrho\right\|_{L^{2}}+\left\|\nabla^{l} u\right\|_{L^{6}}\|\nabla \varrho\|_{L^{3}}\right) \\
& \quad \lesssim \delta\left(\left\|\nabla^{l} \varrho\right\|_{L^{2}}^{2}+\left\|\nabla^{l+1} u\right\|_{L^{2}}^{2}\right) .
\end{aligned}
$$

By the product estimates (A.4) and (4.2), we obtain

$$
\begin{aligned}
& -\int h^{\prime}(\bar{\rho}) \nabla^{l} \varrho \nabla^{l}\left(u \cdot \nabla\left(\rho_{s}-\bar{\rho}\right)\right) \lesssim\left\|\nabla^{l} \varrho\right\|_{L^{2}}\left\|\nabla^{l}\left(u \cdot \nabla\left(\rho_{s}-\bar{\rho}\right)\right)\right\|_{L^{2}} \\
& \quad \lesssim\left\|\nabla^{l} \varrho\right\|_{L^{2}}\left(\|u\|_{L^{\infty}}\left\|\nabla^{l+1}\left(\rho_{s}-\bar{\rho}\right)\right\|_{L^{2}}+\left\|\nabla^{l} u\right\|_{L^{6}}\left\|\nabla\left(\rho_{s}-\bar{\rho}\right)\right\|_{L^{3}}\right) \\
& \quad \lesssim \delta\left(\left\|\nabla^{l} \varrho\right\|_{L^{2}}^{2}+\left\|\nabla^{l+1} u\right\|_{L^{2}}^{2}+\|u\|_{L^{\infty}}^{2}\right)
\end{aligned}
$$

and

$$
\begin{aligned}
& -\int h^{\prime}(\bar{\rho}) \nabla^{l} \varrho \nabla^{l}\left(\left(\varrho+\rho_{s}-\bar{\rho}\right) \operatorname{div} u\right) \lesssim\left\|\nabla^{l} \varrho\right\|_{L^{2}}\left\|\nabla^{l}\left(\left(\varrho+\rho_{s}-\bar{\rho}\right) \operatorname{div} u\right)\right\|_{L^{2}} \\
& \quad \lesssim\left\|\nabla^{l} \varrho\right\|_{L^{2}}\left(\left\|\varrho+\rho_{s}-\bar{\rho}\right\|_{L^{\infty}}\left\|\nabla^{l} \operatorname{div} u\right\|_{L^{2}}+\left\|\nabla^{l} \varrho\right\|_{L^{2}}\|\nabla u\|_{L^{\infty}}+\left\|\nabla^{l}\left(\rho_{s}-\bar{\rho}\right)\right\|_{L^{6}}\|\nabla u\|_{L^{3}}\right) \\
& \quad \lesssim \delta\left(\left\|\nabla^{l} \varrho\right\|_{L^{2}}^{2}+\left\|\nabla^{l+1} u\right\|_{L^{2}}^{2}+\|\nabla u\|_{L^{3}}^{2}\right) .
\end{aligned}
$$

Hence, we may conclude that

$$
-\int h^{\prime}(\bar{\rho}) \nabla^{l} \varrho \nabla^{l} \operatorname{div}\left(\left(\varrho+\rho_{s}-\bar{\rho}\right) u\right) \lesssim \delta\left(\left\|\nabla^{l} \varrho\right\|_{L^{2}}^{2}+\left\|\nabla^{l+1} u\right\|_{L^{2}}^{2}+\|u\|_{L^{\infty}}^{2}+\|\nabla u\|_{L^{3}}^{2}\right) .
$$

Next, by Hölder's and Sobolev's inequalities, the product estimates (A.4) and the interpolation estimates (A.1), we obtain that for $l=0$,

$$
-\int \bar{\rho} \nabla^{l} u \cdot \nabla^{l}(u \cdot \nabla u)=-\int \bar{\rho} u \cdot(u \cdot \nabla u) \lesssim\|u\|_{L^{3}}\|u\|_{L^{6}}\|\nabla u\|_{L^{2}} \lesssim \delta\|\nabla u\|_{L^{2}}^{2}
$$

for $l \geqslant 1$,

$$
\begin{aligned}
& -\int \bar{\rho} \nabla^{l} u \cdot \nabla^{l}(u \cdot \nabla u)=\left\|\nabla^{l} u\right\|_{L^{6}}\left\|\nabla^{l}(u \cdot \nabla u)\right\|_{L^{6 / 5}} \\
& \quad \lesssim\left\|\nabla^{l+1} u\right\|_{L^{2}}\left(\|u\|_{L^{3}}\left\|\nabla^{l+1} u\right\|_{L^{2}}+\left\|\nabla^{l} u\right\|_{L^{2}}\|\nabla u\|_{L^{3}}\right) \\
& \quad \lesssim\left\|\nabla^{l+1} u\right\|_{L^{2}}\left(\|u\|_{L^{3}}\left\|\nabla^{l+1} u\right\|_{L^{2}}+\|u\|_{L^{2}}^{\frac{1}{l+1}}\left\|\nabla^{l+1} u\right\|_{L^{2}}^{\frac{l}{l+1}}\left\|\nabla^{\frac{l+1}{2 l}} u\right\|_{L^{2}}^{\frac{l}{l+1}}\left\|\nabla^{l+1} u\right\|_{L^{2}}^{\frac{1}{l+1}}\right) \\
& \quad \lesssim \delta\left\|\nabla^{l+1} u\right\|_{L^{2}}^{2} .
\end{aligned}
$$


Hence, we may conclude that for $l \geqslant 0$,

$$
-\int \bar{\rho} \nabla^{l} u \cdot \nabla^{l}(u \cdot \nabla u) \lesssim \delta\left\|\nabla^{l+1} u\right\|_{L^{2}}^{2} .
$$

Integrating by parts and by the product estimates (A.4), the nonlinear estimates (A.6) and (4.2), we obtain

$$
\begin{aligned}
& -\int \bar{\rho} \nabla^{l} u \cdot \nabla^{l} \nabla \mathcal{R}=\int \bar{\rho} \nabla^{l} \operatorname{div} u \nabla^{l} \mathcal{R} \lesssim\left\|\nabla^{l+1} u\right\|_{L^{2}}\left\|\nabla^{l} \mathcal{R}\right\|_{L^{2}} \\
& \quad \lesssim \delta\left(\|\varrho\|_{L^{\infty}}^{2}+\left\|\nabla^{l} \varrho\right\|_{L^{2}}^{2}+\left\|\nabla^{l+1} u\right\|_{L^{2}}^{2}\right)
\end{aligned}
$$

and

$$
\begin{aligned}
& -\int \bar{\rho} \nabla^{l} u \cdot \nabla^{l} \nabla\left(\left(h^{\prime}\left(\rho_{s}\right)-h^{\prime}(\bar{\rho})\right) \varrho\right)=\int \bar{\rho} \nabla^{l+1} u \cdot \nabla^{l}\left(\left(h^{\prime}\left(\rho_{s}\right)-h^{\prime}(\bar{\rho})\right) \varrho\right) \\
& \quad \lesssim\left\|\nabla^{l+1} u\right\|_{L^{2}}\left\|\nabla^{l}\left(\left(h^{\prime}\left(\rho_{s}\right)-h^{\prime}(\bar{\rho})\right) \varrho\right)\right\|_{L^{2}} \\
& \quad \lesssim\left\|\nabla^{l+1} u\right\|_{L^{2}}\left(\left\|h^{\prime}\left(\rho_{s}\right)-h^{\prime}(\bar{\rho})\right\|_{L^{\infty}}\left\|\nabla^{l} \varrho\right\|_{L^{2}}+\left\|\nabla^{l}\left(h^{\prime}\left(\rho_{s}\right)-h^{\prime}(\bar{\rho})\right)\right\|_{L^{2}}\|\varrho\|_{L^{\infty}}\right) \\
& \quad \lesssim \delta\left(\left\|\nabla^{l+1} u\right\|_{L^{2}}^{2}+\left\|\nabla^{l} \varrho\right\|_{L^{2}}^{2}+\|\varrho\|_{L^{\infty}}^{2}\right) .
\end{aligned}
$$

We now estimate the last term on the right-hand side of (4.4). For $l=0$, we easily obtain

$$
\begin{aligned}
& \int \bar{\rho} u \cdot\left(\frac{1}{\varrho+\rho_{s}}-\frac{1}{\bar{\rho}}\right)\left(\mu \Delta u+\left(\mu+\mu^{\prime}\right) \nabla \operatorname{div} u\right) \\
& \quad \lesssim\|u\|_{L^{6}}\left\|\frac{1}{\varrho+\rho_{s}}-\frac{1}{\bar{\rho}}\right\|_{L^{2}}\left\|\nabla^{2} u\right\|_{L^{3}} \lesssim \delta\left(\|\nabla u\|_{L^{2}}^{2}+\left\|\nabla^{2} u\right\|_{L^{3}}^{2}\right) .
\end{aligned}
$$

For $l \geqslant 1$, by the integration by parts, the product estimates (A.4) and (4.2), we obtain

$$
\begin{aligned}
\int \bar{\rho} & \nabla^{l} u \cdot \nabla^{l}\left(\left(\frac{1}{\varrho+\rho_{s}}-\frac{1}{\bar{\rho}}\right)\left(\mu \Delta u+\left(\mu+\mu^{\prime}\right) \nabla \operatorname{div} u\right)\right) \\
& =-\int \bar{\rho} \nabla^{l+1} u \cdot \nabla^{l-1}\left(\left(\frac{1}{\varrho+\rho_{s}}-\frac{1}{\bar{\rho}}\right)\left(\mu \Delta u+\left(\mu+\mu^{\prime}\right) \nabla \operatorname{div} u\right)\right) \\
& \lesssim\left\|\nabla^{l+1} u\right\|_{L^{2}}\left\|\nabla^{l-1}\left(\left(\frac{1}{\varrho+\rho_{s}}-\frac{1}{\bar{\rho}}\right)\left(\mu \Delta u+\left(\mu+\mu^{\prime}\right) \nabla \operatorname{div} u\right)\right)\right\|_{L^{2}} \\
& \lesssim\left\|\nabla^{l+1} u\right\|_{L^{2}}\left(\left\|\frac{1}{\varrho+\rho_{s}}-\frac{1}{\bar{\rho}}\right\|_{L^{\infty}}\left\|\nabla^{l+1} u\right\|_{L^{2}}+\left\|\nabla^{l-1}\left(\frac{1}{\varrho+\rho_{s}}-\frac{1}{\bar{\rho}}\right)\right\|_{L^{6}}\left\|\nabla^{2} u\right\|_{L^{3}}\right) \\
& \lesssim \delta\left(\left\|\nabla^{l+1} u\right\|_{L^{2}}^{2}+\left\|\nabla^{2} u\right\|_{L^{3}}^{2}\right) .
\end{aligned}
$$

Hence, we may conclude that for $l \geqslant 0$,

$$
\begin{aligned}
& \int \bar{\rho} \nabla^{l} u \cdot \nabla^{l}\left(\left(\frac{1}{\varrho+\rho_{s}}-\frac{1}{\bar{\rho}}\right)\left(\mu \Delta u+\left(\mu+\mu^{\prime}\right) \nabla \operatorname{div} u\right)\right) \\
& \quad \lesssim \delta\left(\left\|\nabla^{l+1} u\right\|_{L^{2}}^{2}+\left\|\nabla^{2} u\right\|_{L^{3}}^{2}\right) .
\end{aligned}
$$

Now, we turn to estimate the terms on the left-hand side of (4.4). For the second term, we deduce from (1.2) that

$$
\int \mu\left|\nabla^{l+1} u\right|^{2}+\left(\mu+\mu^{\prime}\right)\left|\nabla^{l} \operatorname{div} u\right|^{2} \gtrsim\left\|\nabla^{l+1} u\right\|_{L^{2}}^{2}
$$

For the remaining Poisson term, we integrate by parts and use the first equation and the Poisson equation in (4.1) to obtain 


$$
\begin{aligned}
& -\int \bar{\rho} \nabla^{l} u \cdot \nabla^{l} \nabla \Phi=\int \nabla^{l} \Phi \nabla^{l} \operatorname{div}(\bar{\rho} u) \\
& =-\int \nabla^{l} \Phi \nabla^{l} \partial_{t} \varrho+\nabla^{l} \Phi \nabla^{l} \operatorname{div}\left(\left(\varrho+\rho_{s}-\bar{\rho}\right) u\right) \\
& =-\int \nabla^{l} \Phi \nabla^{l} \partial_{t} \Delta \Phi-\nabla^{l}\left(\left(\varrho+\rho_{s}-\bar{\rho}\right) u\right) \cdot \nabla^{l} \nabla \Phi \\
& =\frac{1}{2} \frac{d}{d t} \int\left|\nabla^{l} \nabla \Phi\right|^{2}+\int \nabla^{l}\left(\left(\varrho+\rho_{s}-\bar{\rho}\right) u\right) \cdot \nabla^{l} \nabla \Phi .
\end{aligned}
$$

By the product estimates (A.4) and (4.2), we obtain

$$
\begin{aligned}
\int & \nabla^{l}\left(\left(\rho_{s}-\bar{\rho}\right) u\right) \cdot \nabla^{l} \nabla \Phi \lesssim\left\|\nabla^{l}\left(\left(\rho_{s}-\bar{\rho}\right) u\right)\right\|_{L^{2}}\left\|\nabla^{l} \nabla \Phi\right\|_{L^{2}} \\
& \lesssim\left(\left\|\rho_{s}-\bar{\rho}\right\|_{L^{\infty}}\left\|\nabla^{l} u\right\|_{L^{2}}+\left\|\nabla^{l}\left(\rho_{s}-\bar{\rho}\right)\right\|_{L^{2}}\|u\|_{L^{\infty}}\right)\left\|\nabla^{l} \nabla \Phi\right\|_{L^{2}} \\
& \lesssim \delta\left(\left\|\nabla^{l} \nabla \Phi\right\|_{L^{2}}^{2}+\left\|\nabla^{l} u\right\|_{L^{2}}^{2}+\|u\|_{L^{\infty}}^{2}\right)
\end{aligned}
$$

and

$$
\begin{aligned}
\int & \nabla^{l}(\varrho u) \cdot \nabla^{l} \nabla \Phi \lesssim\left\|\nabla^{l}(\varrho u)\right\|_{L^{2}}\left\|\nabla^{l} \nabla \Phi\right\|_{L^{2}} \\
& \lesssim\left(\|\varrho\|_{L^{\infty}}\left\|\nabla^{l} u\right\|_{L^{2}}+\left\|\nabla^{l} \varrho\right\|_{L^{2}}\|u\|_{L^{\infty}}\right)\left\|\nabla^{l} \nabla \Phi\right\|_{L^{2}} \\
& \lesssim \delta\left(\left\|\nabla^{l} \nabla \Phi\right\|_{L^{2}}^{2}+\left\|\nabla^{l} u\right\|_{L^{2}}^{2}+\left\|\nabla^{l} \varrho\right\|_{L^{2}}^{2}\right) .
\end{aligned}
$$

Hence, we may conclude that

$$
-\int \bar{\rho} \nabla^{l} u \cdot \nabla^{l} \nabla \Phi \geqslant \frac{1}{2} \frac{d}{d t} \int\left|\nabla^{l} \nabla \Phi\right|^{2}-C \delta\left(\left\|\nabla^{l} \nabla \Phi\right\|_{L^{2}}^{2}+\left\|\nabla^{l} \varrho\right\|_{L^{2}}^{2}+\left\|\nabla^{l} u\right\|_{L^{2}}^{2}+\|u\|_{L^{\infty}}^{2}\right) .
$$

Consequently, plugging the estimates (4.10), (4.13)-(4.15), (4.18)-(4.19) and (4.23) into (4.4), since $\delta$ is small, we deduce (4.3).

We now recover the dissipation estimates for $\varrho$.

Lemma 4.2. For $0 \leqslant l \leqslant k-1$, we have

$$
\begin{aligned}
& \frac{d}{d t} \int \nabla^{l} u \cdot \nabla \nabla^{l} \varrho+\left\|\nabla^{l} \varrho\right\|_{L^{2}}^{2}+\left\|\nabla^{l+1} \varrho\right\|_{L^{2}}^{2} \\
& \quad \lesssim\left\|\nabla^{l+1} u\right\|_{L^{2}}^{2}+\left\|\nabla^{l+2} u\right\|_{L^{2}}^{2}+\delta\left(\|\varrho\|_{L^{\infty}}^{2}+\|u\|_{L^{\infty}}^{2}+\|\nabla u\|_{L^{3}}^{2}+\left\|\nabla^{2} u\right\|_{L^{3}}^{2}\right) .
\end{aligned}
$$

Proof. Applying $\nabla^{l}$ to the second equation in (4.1), multiplying the resulting identity by $\nabla^{l} \nabla \varrho$ and then integrating over $\mathbb{R}^{3}$, we obtain

$$
\begin{aligned}
& \int \nabla^{l} \partial_{t} u \cdot \nabla^{l} \nabla \varrho+\int h^{\prime}(\bar{\rho})\left|\nabla^{l+1} \varrho\right|^{2}-\int \nabla^{l} \nabla \Phi \cdot \nabla^{l} \nabla \varrho \\
& \lesssim\left(\left\|\nabla^{l+1}\left(\left(h^{\prime}\left(\rho_{s}\right)-h^{\prime}(\bar{\rho})\right) \varrho\right)\right\|_{L^{2}}+\left\|\nabla^{l+2} u\right\|_{L^{2}}+\left\|\nabla^{l}(u \cdot \nabla u)\right\|_{L^{2}}\right. \\
&\left.\quad+\left\|\nabla^{l+1} \mathcal{R}\right\|_{L^{2}}+\left\|\nabla^{l}\left(\left(\frac{1}{\varrho+\rho_{s}}-\frac{1}{\bar{\rho}}\right) \nabla^{2} u\right)\right\|_{L^{2}}\right)\left\|\nabla^{l+1} \varrho\right\|_{L^{2}} .
\end{aligned}
$$

As (3.46), we deduce

$$
\begin{aligned}
& -\int \nabla^{l} \partial_{t} u \cdot \nabla \nabla^{l} \varrho=-\frac{d}{d t} \int \nabla^{l} u \cdot \nabla \nabla^{l} \varrho+\int \nabla^{l} u \cdot \nabla \nabla^{l} \partial_{t} \varrho \\
& =-\frac{d}{d t} \int \nabla^{l} u \cdot \nabla \nabla^{l} \varrho+\int \nabla^{l} \operatorname{div} u \nabla^{l}\left(u \cdot \nabla \varrho+\varrho \operatorname{div} u+\rho_{s} \operatorname{div} u+u \cdot \nabla \rho_{s}\right)
\end{aligned}
$$




$$
\begin{aligned}
\leqslant & -\frac{d}{d t} \int \nabla^{l} u \cdot \nabla \nabla^{l} \varrho+\left\|\nabla^{l+1} u\right\|_{L^{2}}^{2}+\left\|\nabla^{l}(u \cdot \nabla \varrho)\right\|_{L^{2}}^{2} \\
& +\left\|\nabla^{l}(\varrho \operatorname{div} u)\right\|_{L^{2}}^{2}+\left\|\nabla^{l}\left(\rho_{s} \operatorname{div} u\right)\right\|_{L^{2}}^{2}+\left\|\nabla^{l}\left(u \cdot \nabla \rho_{s}\right)\right\|_{L^{2}}^{2} .
\end{aligned}
$$

As (3.51)-(3.53), applying the product estimates (A.4) and the nonlinear estimates (A.6) as in Lemma 4.1, we obtain

$$
\begin{aligned}
& \frac{d}{d t} \int \nabla^{l} u \cdot \nabla \nabla^{l} \varrho+\int h^{\prime}\left(\rho_{s}\right)\left|\nabla^{l+1} \varrho\right|^{2}+\left\|\nabla^{l} \varrho\right\|_{L^{2}}^{2} \\
& \lesssim\left(\left\|\nabla^{l+1} u\right\|_{L^{2}}+\left\|\nabla^{l+2} u\right\|_{L^{2}}+\delta\|\varrho\|_{L^{\infty}}+\delta\|u\|_{L^{\infty}}\right. \\
& \left.\quad+\delta\|\nabla u\|_{L^{3}}+\delta\left\|\nabla^{2} u\right\|_{L^{3}}+\delta\left\|\nabla^{l+1} \varrho\right\|_{L^{2}}\right)\left\|\nabla^{l+1} \varrho\right\|_{L^{2}} .
\end{aligned}
$$

By Cauchy's inequality and since $\delta$ is small, we deduce (4.24) from (4.27).

We now combine Lemmas 4.14 .2 to derive the following proposition.

Proposition 4.3. Let $k \geqslant 3$ and $0 \leqslant \ell \leqslant 3 / 2$. Then there exists an energy functional $\mathcal{E}_{\ell}^{k}$ equivalently to $\left\|\nabla^{\ell}(\varrho, u, \nabla \Phi)\right\|_{H^{k-\ell}}^{2}$ such that

$$
\frac{d}{d t} \mathcal{E}_{\ell}^{k}+\left\|\nabla^{\ell} \varrho\right\|_{H^{k-\ell}}^{2}+\left\|\nabla^{\ell+1} u\right\|_{H^{k-\ell}}^{2} \lesssim \delta\left(\left\|\nabla^{\ell} \nabla \Phi\right\|_{L^{2}}^{2}+\left\|\nabla^{\ell} u\right\|_{L^{2}}^{2}+\|\varrho\|_{L^{\infty}}^{2}+\|u\|_{L^{\infty}}^{2}\right) .
$$

Proof. Summing up the estimates (4.3) of Lemma 4.1 for from $l=\ell$ to $k$, by the Poisson equation in (4.1), we obtain

$$
\begin{aligned}
& \frac{d}{d t}\left(h^{\prime}(\bar{\rho})\left\|\nabla^{\ell} \varrho\right\|_{H^{k-\ell}}^{2}+\bar{\rho}\left\|\nabla^{\ell} u\right\|_{H^{k-\ell}}^{2}+\left\|\nabla^{\ell} \nabla \Phi\right\|_{H^{k-\ell}}^{2}\right)+\left\|\nabla^{\ell+1} u\right\|_{H^{k-\ell}}^{2} \\
& \quad \lesssim \delta\left(\left\|\nabla^{\ell} \varrho\right\|_{H^{k-\ell}}^{2}+\left\|\nabla^{\ell} \nabla \Phi\right\|_{L^{2}}^{2}+\left\|\nabla^{\ell} u\right\|_{H^{k-\ell}}^{2}+\|\varrho\|_{L^{\infty}}^{2}+\|u\|_{L^{\infty}}^{2}+\|\nabla u\|_{L^{3}}^{2}+\left\|\nabla^{2} u\right\|_{L^{3}}^{2}\right) .
\end{aligned}
$$

Summing up the estimates (4.24) of Lemma 4.2 for from $l=\ell$ to $k-1$, we obtain

$$
\begin{aligned}
& \frac{d}{d t} \sum_{\ell \leqslant l \leqslant k-1} \int \nabla^{l} u \cdot \nabla \nabla^{l} \varrho+\left\|\nabla^{\ell} \varrho\right\|_{H^{k-\ell}}^{2} \\
& \quad \lesssim\left\|\nabla^{\ell+1} u\right\|_{H^{k-\ell}}^{2}+\delta\left(\|\varrho\|_{L^{\infty}}^{2}+\|u\|_{L^{\infty}}^{2}+\|\nabla u\|_{L^{3}}^{2}+\left\|\nabla^{2} u\right\|_{L^{3}}^{2}\right) .
\end{aligned}
$$

Multiplying (4.30) by a small constant $\epsilon>0$ and then adding the resulting inequality to (4.29), since $\delta$ is small, we deduce that for $0 \leqslant \ell \leqslant k-1$,

$$
\begin{aligned}
& \frac{d}{d t}\left(h^{\prime}(\bar{\rho})\left\|\nabla^{\ell} \varrho\right\|_{H^{k-\ell}}^{2}+\bar{\rho}\left\|\nabla^{\ell} u\right\|_{H^{k-\ell}}^{2}+\left\|\nabla^{\ell} \nabla \Phi\right\|_{H^{k-\ell}}^{2}+\epsilon \sum_{\ell \leqslant l \leqslant k-1} \int \nabla^{l} u \cdot \nabla \nabla^{l} \varrho\right) \\
& \quad+\left\|\nabla^{\ell} \varrho\right\|_{H^{k-\ell}}^{2}+\left\|\nabla^{\ell+1} u\right\|_{H^{k-\ell}}^{2} \\
& \quad \lesssim \delta\left(\left\|\nabla^{\ell} \nabla \Phi\right\|_{L^{2}}^{2}+\left\|\nabla^{\ell} u\right\|_{L^{2}}^{2}+\|\varrho\|_{L^{\infty}}^{2}+\|u\|_{L^{\infty}}^{2}+\|\nabla u\|_{L^{3}}^{2}+\left\|\nabla^{2} u\right\|_{L^{3}}^{2}\right) .
\end{aligned}
$$

We define $\mathcal{E}_{\ell}^{k}$ to be the expression under the time derivative in (4.31). Since $\epsilon$ is small, $\mathcal{E}_{\ell}^{k}$ is equivalent to $\left\|\nabla^{\ell}(\varrho, u, \nabla \Phi)\right\|_{H^{k-\ell}}^{2}$. Then we deduce that for $0 \leqslant \ell \leqslant k-1$,

$$
\begin{aligned}
& \frac{d}{d t} \mathcal{E}_{\ell}^{k}+\left\|\nabla^{\ell} \varrho\right\|_{H^{k-\ell}}^{2}+\left\|\nabla^{\ell+1} u\right\|_{H^{k-\ell}}^{2} \\
& \quad \lesssim \delta\left(\left\|\nabla^{\ell} \nabla \Phi\right\|_{L^{2}}^{2}+\left\|\nabla^{\ell} u\right\|_{L^{2}}^{2}+\|\varrho\|_{L^{\infty}}^{2}+\|u\|_{L^{\infty}}^{2}+\|\nabla u\|_{L^{3}}^{2}+\left\|\nabla^{2} u\right\|_{L^{3}}^{2}\right) .
\end{aligned}
$$


Now we take $0 \leqslant \ell \leqslant 3 / 2$. Then by the interpolation, we have

$$
\|\nabla u\|_{L^{3}}^{2} \lesssim\left\|\nabla^{3 / 2} u\right\|_{L^{2}}^{2} \lesssim\left\|\nabla^{\ell} u\right\|_{L^{2}}^{2}+\left\|\nabla^{k} u\right\|_{L^{2}}^{2}
$$

and

$$
\left\|\nabla^{2} u\right\|_{L^{3}}^{2} \lesssim\left\|\nabla^{5 / 2} u\right\|_{L^{2}}^{2} \lesssim\left\|\nabla^{\ell+1} u\right\|_{L^{2}}^{2}+\left\|\nabla^{k} u\right\|_{L^{2}}^{2} .
$$

Since $\delta$ is small, (4.32) implies (4.28) by (4.33)-(4.34).

4.2. Duhamel form analysis. In order to use the linear decay estimates for the linear system with constant coefficients, we will rewrite the Navier-Stokes-Poisson system (4.1) as the NavierStokes equations with a non-local self-consistent force in the following form:

$$
\left\{\begin{array}{l}
\partial_{t} \varrho+\bar{\rho} \operatorname{div} u=N^{1}, \\
\partial_{t} u+h^{\prime}(\bar{\rho}) \nabla \varrho-\frac{1}{\bar{\rho}}\left(\mu \Delta u+\left(\mu+\mu^{\prime}\right) \nabla \operatorname{div} u\right)-\nabla \Delta^{-1} \varrho=N^{2}, \\
\left.(\varrho, u)\right|_{t=0}=\left(\varrho_{0}, u_{0}\right),
\end{array}\right.
$$

where the "nonlinear" terms are given by

$$
N^{1}=-\operatorname{div}\left(\left(\varrho+\rho_{s}-\bar{\rho}\right) u\right)
$$

and

$$
N^{2}=-u \cdot \nabla u-\nabla \mathcal{R}-\nabla\left(\left(h^{\prime}\left(\rho_{s}\right)-h^{\prime}(\bar{\rho})\right) \varrho\right)+\left(\frac{1}{\varrho+\rho_{s}}-\frac{1}{\bar{\rho}}\right)\left(\mu \Delta u+\left(\mu+\mu^{\prime}\right) \nabla \operatorname{div} u\right) .
$$

By the Duhamel principle, the solution $(\varrho, u)$ to the problem (4.35) can be expressed as

$$
(\varrho, u)(t)=e^{-t \mathbb{A}}\left(\varrho_{0}, u_{0}\right)+\int_{0}^{t} e^{-(t-\tau) \mathbb{A}}\left(N^{1}, N^{2}\right)(\tau) d \tau .
$$

Here the matrix differential operator $\mathbb{A}$ is defined by

$$
\mathbb{A}=\left(\begin{array}{cc}
0 & \bar{\rho} \operatorname{div} \\
h^{\prime}(\bar{\rho}) \nabla-\nabla \Delta^{-1} & -\frac{1}{\bar{\rho}}\left(\mu \Delta+\left(\mu+\mu^{\prime}\right) \nabla \operatorname{div}\right)
\end{array}\right) .
$$

In light of the analysis in [10] and [16], we have the followings about the time decay rates of the solution semigroup $e^{-t \mathbb{A}}$ of the linearized system of (4.35).

Lemma 4.4. Let $(\widetilde{\varrho}, \widetilde{u})=e^{-t \mathbb{A}}\left(\varrho_{0}, u_{0}\right)$. Then for $1 \leqslant p \leqslant 2, q \geqslant 2$ and $\ell \geqslant 0$, we have

$$
\left\|\nabla^{\ell} \varrho\right\|_{L^{q}} \lesssim(1+t)^{-\frac{3}{2}\left(\frac{1}{p}-\frac{1}{q}\right)-\frac{\ell}{2}-\frac{1}{2}}\left(\left\|\left(\nabla^{-1} \varrho_{0}, u_{0}\right)\right\|_{L^{p}}+\left\|\nabla^{\ell}\left(\varrho_{0}, u_{0}\right)\right\|_{L^{q}}\right)
$$

and

$$
\left\|\nabla^{\ell} \widetilde{u}\right\|_{L^{q}} \lesssim(1+t)^{-\frac{3}{2}\left(\frac{1}{p}-\frac{1}{q}\right)-\frac{\ell}{2}}\left(\left\|\left(\nabla^{-1} \varrho_{0}, u_{0}\right)\right\|_{L^{p}}+\left\|\nabla^{\ell}\left(\varrho_{0}, u_{0}\right)\right\|_{L^{q}}\right) .
$$

Applying Lemma 4.4 to (4.38), we obtain the following proposition.

Proposition 4.5. It holds that for $1 \leqslant p, r \leqslant 2, q \geqslant 2$ and $\ell \geqslant 0$,

$$
\begin{aligned}
\left\|\nabla^{\ell} \varrho(t)\right\|_{L^{q}} \lesssim & (1+t)^{-\frac{3}{2}\left(\frac{1}{p}-\frac{1}{q}\right)-\frac{\ell}{2}-\frac{1}{2}}\left(\left\|\left(\nabla^{-1} \varrho_{0}, u_{0}\right)\right\|_{L^{p}}+\left\|\nabla^{\ell}\left(\varrho_{0}, u_{0}\right)\right\|_{L^{q}}\right) \\
& +\int_{0}^{t}(1+t-\tau)^{-\frac{3}{2}\left(\frac{1}{r}-\frac{1}{q}\right)-\frac{\ell}{2}-\frac{1}{2}}\left(\left\|\left(\nabla^{-1} N^{1}, N^{2}\right)(\tau)\right\|_{L^{r}}+\left\|\nabla^{\ell}\left(N^{1}, N^{2}\right)(\tau)\right\|_{L^{q}}\right) d \tau
\end{aligned}
$$

and

$$
\left\|\nabla^{\ell} u(t)\right\|_{L^{q}} \lesssim(1+t)^{-\frac{3}{2}\left(\frac{1}{p}-\frac{1}{q}\right)-\frac{\ell}{2}}\left(\left\|\left(\nabla^{-1} \varrho_{0}, u_{0}\right)\right\|_{L^{p}}+\left\|\nabla^{\ell}\left(\varrho_{0}, u_{0}\right)\right\|_{L^{q}}\right)
$$




$$
+\int_{0}^{t}(1+t-\tau)^{-\frac{3}{2}\left(\frac{1}{r}-\frac{1}{q}\right)-\frac{\ell}{2}}\left(\left\|\left(\nabla^{-1} N^{1}, N^{2}\right)(\tau)\right\|_{L^{r}}+\left\|\nabla^{\ell}\left(N^{1}, N^{2}\right)(\tau)\right\|_{L^{q}}\right) d \tau
$$

Finally, we record the following estimates of nonlinear terms appeared in (4.42)-(4.43).

Lemma 4.6. It holds that for $1<r \leqslant 2$,

$$
\begin{aligned}
\left\|\left(\nabla^{-1} N^{1}, N^{2}\right)\right\|_{L^{r}} \lesssim & \left(\delta+\left\|\rho_{s}-\bar{\rho}\right\|_{W^{1, r}}\right)\left(\|u\|_{L^{\infty}}+\|\nabla \varrho\|_{H^{2}}+\left\|\nabla^{2} u\right\|_{H^{1}}\right) \\
& +\|\varrho\|_{L^{2}}\left\|\nabla^{3-\frac{3}{r}} u\right\|_{L^{2}}+\|u\|_{L^{2}}\left\|\nabla^{4-\frac{3}{r}} u\right\|_{L^{2}} ;
\end{aligned}
$$

for $0 \leqslant \ell \leqslant 3 / 2$,

$$
\left\|\nabla^{\ell}\left(N^{1}, N^{2}\right)\right\|_{L^{2}} \lesssim \delta\left(\|u\|_{L^{\infty}}+\|\nabla \varrho\|_{H^{2}}+\left\|\nabla^{2} u\right\|_{H^{2}}\right)
$$

and

$$
\left\|\left(N^{1}, N^{2}\right)\right\|_{L^{\infty}} \lesssim \delta\left(\|u\|_{L^{\infty}}+\|\nabla \varrho\|_{H^{2}}+\left\|\nabla^{2} u\right\|_{H^{2}}\right) .
$$

Proof. We will estimate the nonlinear terms term by term. First, for $1<r \leqslant 2$, by the singular integral theory [14, the identity (A.9) in the proof of Lemma A.3 and Hölder's and Sobolev's inequalities, we obtain

$$
\begin{aligned}
& \left\|\nabla^{-1} \operatorname{div}\left(\left(\varrho+\rho_{s}-\bar{\rho}\right) u\right)\right\|_{L^{r}} \lesssim\left\|\left(\varrho+\rho_{s}-\bar{\rho}\right) u\right\|_{L^{r}} \\
& \quad \lesssim\|\varrho\|_{L^{2}}\|u\|_{L^{1 / r-1 / 2}}+\left\|\rho_{s}-\bar{\rho}\right\|_{L^{r}}\|u\|_{L^{\infty}} \lesssim\|\varrho\|_{L^{2}}\left\|\nabla^{3-\frac{3}{r}} u\right\|_{L^{2}}+\left\|\rho_{s}-\bar{\rho}\right\|_{L^{r}}\|u\|_{L^{\infty}} ; \\
& \|u \cdot \nabla u\|_{L^{r}} \lesssim\|u\|_{L^{2}}\|\nabla u\|_{L^{1 / r-1 / 2}} \lesssim\|u\|_{L^{2}}\left\|\nabla^{4-\frac{3}{r}} u\right\|_{L^{2}} ; \\
& \quad\|\nabla \mathcal{R}\|_{L^{r}} \lesssim\left\|h^{\prime \prime}\left(\rho_{s}\right) \varrho \nabla \varrho\right\|_{L^{r}}+\left\|\mathcal{R}\left(h^{\prime}\right)\left(\nabla \varrho+\nabla \rho_{s}\right)\right\|_{L^{r}} \\
& \quad \lesssim\|\varrho\|_{L^{1 / r-1 / 2}}\|\nabla \varrho\|_{L^{2}}+\|\varrho\|_{L^{1 / r-1 / 2}}\|\varrho\|_{L^{\infty}}\left(\|\nabla \varrho\|_{L^{2}}+\left\|\nabla \rho_{s}\right\|_{L^{2}}\right) \lesssim \delta\|\nabla \varrho\|_{H^{2}} ; \\
& \quad\left\|\nabla\left(\left(h^{\prime}\left(\rho_{s}\right)-h^{\prime}(\bar{\rho})\right) \varrho\right)\right\|_{L^{r}} \lesssim\left\|h^{\prime}\left(\rho_{s}\right)-h^{\prime}(\bar{\rho})\right\|_{L^{r}}\|\nabla \varrho\|_{L^{\infty}}+\left\|\nabla\left(h^{\prime}\left(\rho_{s}\right)-h^{\prime}(\bar{\rho})\right)\right\|_{L^{r}}\|\varrho\|_{L^{\infty}} \\
& \quad \lesssim\left\|\rho_{s}-\bar{\rho}\right\|_{W^{1, r}}\|\nabla \varrho\|_{H^{2}} ; \| \\
& \left\|\left(\frac{1}{\varrho+\rho_{s}}-\frac{1}{\bar{\rho}}\right) \nabla^{2} u\right\|_{L^{r}} \lesssim\left\|\frac{1}{\varrho+\rho_{s}}-\frac{1}{\bar{\rho}}\right\|_{L^{2}}\left\|\nabla^{2} u\right\|_{L^{1 / r-1 / 2}} \lesssim \delta\left\|\nabla^{2} u\right\|_{H^{1}} \cdot
\end{aligned}
$$

These estimates (4.47)-(4.51) give (4.44).

Next, for $0 \leqslant \ell \leqslant 3 / 2$, by the product estimates (A.4), the nonlinear estimates (A.5) and Sobolev's inequality, we obtain

$$
\begin{aligned}
& \left\|\nabla^{\ell} \operatorname{div}\left(\left(\varrho+\rho_{s}-\bar{\rho}\right) u\right)\right\|_{L^{2}} \lesssim\left\|\varrho+\rho_{s}-\bar{\rho}\right\|_{L^{3}}\left\|\nabla^{\ell+1} u\right\|_{L^{6}}+\left\|\nabla^{\ell+1}\left(\varrho+\rho_{s}-\bar{\rho}\right)\right\|_{L^{2}}\|u\|_{L^{\infty}} \\
& \quad \lesssim \delta\left(\|u\|_{L^{\infty}}+\left\|\nabla^{2} u\right\|_{H^{2}}\right) ; \\
& \left\|\nabla^{\ell}(u \cdot \nabla u)\right\|_{L^{2}} \lesssim\left\|\nabla^{\ell} u\right\|_{L^{3}}\|\nabla u\|_{L^{6}}+\|u\|_{L^{3}}\left\|\nabla^{\ell+1} u\right\|_{L^{6}} \lesssim \delta\left\|\nabla^{2} u\right\|_{H^{2}} \\
& \left\|\nabla^{\ell} \nabla \mathcal{R}\right\|_{L^{2}} \lesssim \delta\left(\|\nabla \varrho\|_{L^{2}}+\left\|\nabla^{\ell+1} \varrho\right\|_{L^{2}}\right) \lesssim \delta\|\nabla \varrho\|_{H^{2}} ; \\
& \left\|\nabla^{\ell} \nabla\left(\left(h^{\prime}\left(\rho_{s}\right)-h^{\prime}(\bar{\rho})\right) \varrho\right)\right\|_{L^{2}} \\
& \quad \lesssim\left\|\nabla^{\ell+1}\left(h^{\prime}\left(\rho_{s}\right)-h^{\prime}(\bar{\rho})\right)\right\|_{L^{3}}\|\varrho\|_{L^{6}}+\left\|h^{\prime}\left(\rho_{s}\right)-h^{\prime}(\bar{\rho})\right\|_{L^{\infty}}\left\|\nabla^{\ell+1} \varrho\right\|_{L^{2}} \lesssim \delta\|\nabla \varrho\|_{H^{2}} \\
& \left\|\nabla^{\ell}\left(\left(\frac{1}{\varrho+\rho_{s}}-\frac{1}{\bar{\rho}}\right) \nabla^{2} u\right)\right\|_{L^{2}} \\
& \quad \lesssim\left\|\nabla^{\ell}\left(\frac{1}{\varrho+\rho_{s}}-\frac{1}{\bar{\rho}}\right)\right\|_{L^{\infty}}\left\|\nabla^{2} u\right\|_{L^{2}}+\left\|\frac{1}{\varrho+\rho_{s}}-\frac{1}{\bar{\rho}}\right\|_{L^{\infty}}\left\|\nabla^{\ell+2} u\right\|_{L^{2}} \lesssim \delta\left\|\nabla^{2} u\right\|_{H^{2}}
\end{aligned}
$$

These estimates (4.52)-(4.56) yield (4.45).

Note that the estimate (4.46) can be obtained in a similar way. 
4.3. Proof of Theorem 1.3. In this subsection, we will prove Theorem 1.3, Let $k \geqslant 3$ and $0 \leqslant \ell \leqslant 3 / 2$. Adding $\left\|\nabla^{\ell}(u, \nabla \Phi)\right\|_{L^{2}}^{2}$ to both sides of the estimates (4.28) of Proposition 4.3, we obtain

$$
\begin{gathered}
\frac{d}{d t} \mathcal{E}_{\ell}^{k}+\left\|\nabla^{\ell} \varrho\right\|_{H^{k-\ell}}^{2}+\left\|\nabla^{\ell} u\right\|_{H^{k-\ell+1}}^{2}+\left\|\nabla^{\ell} \nabla \Phi\right\|_{L^{2}}^{2} \\
\lesssim\left\|\nabla^{\ell} \nabla \Phi\right\|_{L^{2}}^{2}+\left\|\nabla^{\ell} u\right\|_{L^{2}}^{2}+\|\varrho\|_{L^{\infty}}^{2}+\|u\|_{L^{\infty}}^{2} .
\end{gathered}
$$

Note that $\left\|\nabla^{\ell} \nabla \Phi\right\|_{L^{2}}^{2}$ is equivalent to $\left\|\nabla^{\ell-1} \varrho\right\|_{L^{2}}^{2}$. Hence, we deduce from (4.57) that

$$
\frac{d}{d t} \mathcal{E}_{\ell}^{k}+\lambda \mathcal{E}_{\ell}^{k} \lesssim\left\|\nabla^{\ell-1} \varrho\right\|_{L^{2}}^{2}+\left\|\nabla^{\ell} u\right\|_{L^{2}}^{2}+\|\varrho\|_{L^{\infty}}^{2}+\|u\|_{L^{\infty}}^{2}
$$

for some constant $\lambda>0$. By the Gronwall inequality, we obtain

$$
\begin{aligned}
\| \nabla^{\ell-1} \varrho(t) & \left\|_{H^{k+1-\ell}}^{2}+\right\| \nabla^{\ell} u(t) \|_{H^{k-\ell}}^{2} \lesssim e^{-\lambda t}\left(\left\|\nabla^{\ell}\left(\varrho_{0}, u_{0}\right)\right\|_{H^{k-\ell}}^{2}+\left\|\nabla \Phi_{0}\right\|_{L^{2}}^{2}\right) \\
& +\int_{0}^{t} e^{-\lambda(t-\tau)}\left(\left\|\nabla^{\ell-1} \varrho(\tau)\right\|_{L^{2}}^{2}+\left\|\nabla^{\ell} u(\tau)\right\|_{L^{2}}^{2}+\|\varrho(\tau)\|_{L^{\infty}}^{2}+\|u(\tau)\|_{L^{\infty}}^{2}\right) d \tau
\end{aligned}
$$

We now prove (1.9)-(1.11). So we let $k \geqslant 4,1<r<3 / 2$ and $1 \leqslant p<3 / 2$. For simplicity of notations, we denote

$$
K_{0}:=\left\|\left(\nabla^{-1} \varrho_{0}, u_{0}\right)\right\|_{L^{p}}+\left\|\left(\varrho_{0}, u_{0}\right)\right\|_{H^{k}}+\left\|\nabla \Phi_{0}\right\|_{L^{2}},
$$

and we define

$$
\zeta:=\frac{3}{2}\left(\frac{1}{\max \{p, r\}}-\frac{1}{2}\right) \text { and } \tilde{\delta}:=\delta+\left\|\rho_{s}-\bar{\rho}\right\|_{W^{1, r}} .
$$

It turns out that we have to distinguish our arguments by the value of $r$.

Case 1: $6 / 5 \leqslant r<3 / 2$. In this case, we define

$$
\begin{gathered}
\mathcal{L}(t):=\left\|\nabla^{1 / 2} \varrho(t)\right\|_{L^{2}}+\left\|\nabla^{3 / 2} u(t)\right\|_{L^{2}}+\|\varrho(t)\|_{L^{\infty}}+\|u(t)\|_{L^{\infty}}, \\
\mathcal{M}(t):=\left\|\nabla^{1 / 2} \varrho(t)\right\|_{H^{k-1 / 2}}+\left\|\nabla^{3 / 2} u(t)\right\|_{H^{k-3 / 2}},
\end{gathered}
$$

and

$$
\mathcal{N}(t):=\sup _{0 \leqslant \tau \leqslant t}\left((1+\tau)^{\zeta+\frac{3}{4}}(\mathcal{L}(\tau)+\mathcal{M}(\tau))+(1+\tau)^{\zeta+\frac{1}{2}}\|\varrho(\tau)\|_{L^{2}}+(1+\tau)^{\zeta}\|u(\tau)\|_{L^{2}}\right)
$$

We take $\ell=3 / 2$ in (4.59) to have, in view of (4.62)-(4.63),

$$
\mathcal{M}^{2}(t) \lesssim e^{-\lambda t} K_{0}^{2}+\int_{0}^{t} e^{-\lambda(t-\tau)} \mathcal{L}^{2}(\tau) d \tau
$$

We now estimate the time decay rates of $\mathcal{L}(t)$ by applying the linear decay estimates. By the estimates (4.42) with $\ell=1 / 2$ and $q=2$ of Proposition 4.5] and using the nonlinear estimates (4.44)-(4.45), in view of (4.62)-(4.63), we obtain

$$
\begin{aligned}
& \left\|\nabla^{1 / 2} \varrho(t)\right\|_{L^{2}} \lesssim(1+t)^{-\frac{3}{2}\left(\frac{1}{p}-\frac{1}{2}\right)-\frac{3}{4}} K_{0}+\int_{0}^{t}(1+t-\tau)^{-\frac{3}{2}\left(\frac{1}{r}-\frac{1}{2}\right)-\frac{3}{4}} \tilde{\delta}(\mathcal{L}+\mathcal{M})(\tau) d \tau \\
& +\int_{0}^{t}(1+t-\tau)^{-\frac{3}{2}\left(\frac{1}{r}-\frac{1}{2}\right)-\frac{3}{4}}\left(\|\varrho(\tau)\|_{L^{2}}\left\|\nabla^{3-\frac{3}{r}} u(\tau)\right\|_{L^{2}}+\|u(\tau)\|_{L^{2}}\left\|\nabla^{4-\frac{3}{r}} u(\tau)\right\|_{L^{2}}\right) d \tau
\end{aligned}
$$

By the interpolation, in view of (4.64), we estimate

$$
\begin{aligned}
& \|\varrho(\tau)\|_{L^{2}}\left\|\nabla^{3-\frac{3}{r}} u(\tau)\right\|_{L^{2}}+\|u(\tau)\|_{L^{2}}\left\|\nabla^{4-\frac{3}{r}} u(\tau)\right\|_{L^{2}} \\
& \quad \lesssim\left\|\nabla^{\vartheta} \varrho(\tau)\right\|_{L^{2}}^{2-\frac{2}{r}}\left\|\nabla^{1 / 2} \varrho(\tau)\right\|_{L^{2}}^{\frac{2}{r}-1}\|u(\tau)\|_{L^{2}}^{\frac{2}{r}-1}\left\|\nabla^{3 / 2} u(\tau)\right\|_{L^{2}}^{2-\frac{2}{r}}+\|u(\tau)\|_{L^{2}}\left\|\nabla^{4-\frac{3}{r}} u(\tau)\right\|_{L^{2}} \\
& \quad \lesssim \delta^{2-\frac{2}{r}}(1+\tau)^{\left(-\zeta-\frac{3}{4}\right)\left(\frac{2}{r}-1\right)} \mathcal{N}(t)^{\frac{2}{r}-1} \delta^{\frac{2}{r}-1}(1+\tau)^{\left(-\zeta-\frac{3}{4}\right)\left(2-\frac{2}{r}\right)} \mathcal{N}(t)^{2-\frac{2}{r}}+\delta(1+\tau)^{-\zeta-\frac{3}{4}} \mathcal{N}(t)
\end{aligned}
$$




$$
\lesssim \delta(1+\tau)^{-\zeta-\frac{3}{4}} \mathcal{N}(t)
$$

Here we have used the facts that $\vartheta=(r-2) /(4 r-4) \geqslant-1$ and $4-3 / r \geqslant 3 / 2$ since $r \geqslant 6 / 5$. Hence, plugging the estimates (4.67) into (4.66), in view of (4.64), we have

$$
\begin{aligned}
\left\|\nabla^{1 / 2} \varrho(t)\right\|_{L^{2}} & \lesssim(1+t)^{-\frac{3}{2}\left(\frac{1}{p}-\frac{1}{2}\right)-\frac{3}{4}} K_{0}+\tilde{\delta} \int_{0}^{t}(1+t-\tau)^{-\frac{3}{2}\left(\frac{1}{r}-\frac{1}{2}\right)-\frac{3}{4}}(1+\tau)^{-\zeta-\frac{3}{4}} \mathcal{N}(t) d \tau \\
& \lesssim(1+t)^{-\zeta-\frac{3}{4}}\left(K_{0}+\tilde{\delta} \mathcal{N}(t)\right) .
\end{aligned}
$$

Here we have used the fact $\frac{3}{2}\left(\frac{1}{r}-\frac{1}{2}\right)+\frac{3}{4}>1$ since $r<\frac{3}{2}$. Similarly, by the estimates (4.43) with $\ell=3 / 2$ and $q=2$, and (4.42) (4.43) with $\ell=0$ and $q=\infty$ of Proposition 4.5 respectively, using the nonlinear estimates (4.44)-(4.46), we deduce

$$
\left\|\nabla^{3 / 2} u(t)\right\|_{L^{2}}+\|\varrho(t)\|_{L^{\infty}}+\|u(t)\|_{L^{\infty}} \lesssim(1+t)^{-\zeta-\frac{3}{4}}\left(K_{0}+\tilde{\delta} \mathcal{N}(t)\right) .
$$

We thus deduce from (4.68)-(4.69) that

$$
\mathcal{L}(t) \lesssim(1+t)^{-\zeta-\frac{3}{4}}\left(K_{0}+\tilde{\delta} \mathcal{N}(t)\right)
$$

Now we substitute (4.70) into (4.65) to obtain

$$
\begin{aligned}
\mathcal{M}^{2}(t) & \lesssim e^{-\lambda t} K_{0}^{2}+\int_{0}^{t} e^{-\lambda(t-\tau)}(1+\tau)^{-2 \zeta-\frac{3}{2}}\left(K_{0}^{2}+\tilde{\delta}^{2} \mathcal{N}^{2}(t)\right) d \tau . \\
& \lesssim(1+t)^{-2 \zeta-\frac{3}{2}}\left(K_{0}^{2}+\tilde{\delta}^{2} \mathcal{N}^{2}(t)\right) .
\end{aligned}
$$

Finally, by the estimates (4.42) -(4.43) with $\ell=0$ and $q=2$ of Proposition 4.5, using the estimates (4.44) - (4.45) with $\ell=0$ and (4.67), in view of (4.64), we obtain

$$
\begin{aligned}
\|\varrho(t)\|_{L^{2}} & \lesssim(1+t)^{-\frac{3}{2}\left(\frac{1}{p}-\frac{1}{2}\right)-\frac{1}{2}} K_{0}+\tilde{\delta} \int_{0}^{t}(1+t-\tau)^{-\frac{3}{2}\left(\frac{1}{r}-\frac{1}{2}\right)-\frac{1}{2}}(1+\tau)^{-\zeta-\frac{3}{4}} \mathcal{N}(t) d \tau \\
& \lesssim(1+t)^{-\zeta-\frac{1}{2}}\left(K_{0}+\tilde{\delta} \mathcal{N}(t)\right)
\end{aligned}
$$

and

$$
\begin{aligned}
\|u(t)\|_{L^{2}} & \lesssim(1+t)^{-\frac{3}{2}\left(\frac{1}{p}-\frac{1}{2}\right)} K_{0}+\tilde{\delta} \int_{0}^{t}(1+t-\tau)^{-\frac{3}{2}\left(\frac{1}{r}-\frac{1}{2}\right)}(1+\tau)^{-\zeta-\frac{3}{4}} \mathcal{N}(t) d \tau \\
& \lesssim(1+t)^{-\zeta}\left(K_{0}+\tilde{\delta} \mathcal{N}(t)\right) .
\end{aligned}
$$

Note that we have used the fact $\zeta+\frac{3}{4}>1$ since $p, r<3 / 2$ so that

$$
\int_{0}^{t}(1+t-\tau)^{-\frac{3}{2}\left(\frac{1}{r}-\frac{1}{2}\right)-\frac{1}{2}}(1+\tau)^{-\zeta-\frac{3}{4}} d \tau \lesssim(1+t)^{-\frac{3}{2}\left(\frac{1}{r}-\frac{1}{2}\right)-\frac{1}{2}}
$$

and

$$
\int_{0}^{t}(1+t-\tau)^{-\frac{3}{2}\left(\frac{1}{r}-\frac{1}{2}\right)}(1+\tau)^{-\zeta-\frac{3}{4}} d \tau \lesssim(1+t)^{-\frac{3}{2}\left(\frac{1}{r}-\frac{1}{2}\right)}
$$

By the definition (4.64) of $\mathcal{N}(t)$, we deduce from (4.70) (4.73) that

$$
\mathcal{N}(t) \lesssim K_{0}+\tilde{\delta} \mathcal{N}(t)
$$

This implies

$$
\mathcal{N}(t) \lesssim K_{0}
$$

since $\tilde{\delta}$ is small by Proposition 2.1. This in turn together with the interpolation gives (1.9)-(1.11) for $6 / 5 \leqslant r<3 / 2$ by taking $C_{0}=K_{0}$.

Case 2: $1<r<6 / 5$. In this case, we define

$$
\begin{gathered}
\mathcal{H}(t):=\|\varrho(t)\|_{L^{2}}+\|\nabla u(t)\|_{L^{2}}+\|\varrho(t)\|_{L^{\infty}}+\|u(t)\|_{L^{\infty}}, \\
\mathcal{J}(t):=\|\varrho(t)\|_{H^{k}}+\|\nabla u(t)\|_{H^{k-1}},
\end{gathered}
$$


and

$$
\mathcal{K}(t):=\sup _{0 \leqslant \tau \leqslant t}\left((1+\tau)^{\zeta+\frac{1}{2}}(\mathcal{H}(\tau)+\mathcal{J}(\tau))\right) .
$$

We take $\ell=1$ in (4.59) to have, in view of (4.78)- (4.79),

$$
\mathcal{J}^{2}(t) \lesssim e^{-\lambda t} K_{0}^{2}+\int_{0}^{t} e^{-\lambda(t-\tau)} \mathcal{H}^{2}(\tau) d \tau
$$

We now estimate the time decay rates of $\mathcal{H}(t)$ by applying the linear decay estimates. By the estimates (4.42) with $\ell=0$ and $q=2$ of Proposition 4.5 and using the nonlinear estimates (4.44) -(4.45), in view of (4.78)-(4.79), we obtain

$$
\begin{aligned}
& \|\varrho(t)\|_{L^{2}} \lesssim(1+t)^{-\frac{3}{2}\left(\frac{1}{p}-\frac{1}{2}\right)-\frac{1}{2}} K_{0}+\int_{0}^{t}(1+t-\tau)^{-\frac{3}{2}\left(\frac{1}{r}-\frac{1}{2}\right)-\frac{1}{2}} \tilde{\delta}(\mathcal{H}+\mathcal{J})(\tau) d \tau \\
& +\int_{0}^{t}(1+t-\tau)^{-\frac{3}{2}\left(\frac{1}{r}-\frac{1}{2}\right)-\frac{1}{2}}\left(\|\varrho(\tau)\|_{L^{2}}\left\|\nabla^{3-\frac{3}{r}} u(\tau)\right\|_{L^{2}}+\|u(\tau)\|_{L^{2}}\left\|\nabla^{4-\frac{3}{r}} u(\tau)\right\|_{L^{2}}\right) d \tau .
\end{aligned}
$$

Note that

$$
\|\varrho\|_{L^{2}}\left\|\nabla^{3-\frac{3}{r}} u\right\|_{L^{2}}+\|u\|_{L^{2}}\left\|\nabla^{4-\frac{3}{r}} u\right\|_{L^{2}} \lesssim \delta\left(\|\varrho\|_{L^{2}}+\|\nabla u\|_{H^{1}}\right) \lesssim \delta \mathcal{J} .
$$

Hence, we have

$$
\begin{aligned}
\|\varrho(t)\|_{L^{2}} & \lesssim(1+t)^{-\frac{3}{2}\left(\frac{1}{p}-\frac{1}{2}\right)-\frac{1}{2}} K_{0}+\int_{0}^{t}(1+t-\tau)^{-\frac{3}{2}\left(\frac{1}{r}-\frac{1}{2}\right)-\frac{1}{2}} \tilde{\delta}(\mathcal{H}+\mathcal{J})(\tau) d \tau \\
& \lesssim(1+t)^{-\frac{3}{2}\left(\frac{1}{p}-\frac{1}{2}\right)-\frac{1}{2}} K_{0}+\tilde{\delta} \int_{0}^{t}(1+t-\tau)^{-\frac{3}{2}\left(\frac{1}{r}-\frac{1}{2}\right)-\frac{1}{2}}(1+\tau)^{-\zeta-\frac{1}{2}} \mathcal{K}(t) d \tau \\
& \lesssim(1+t)^{-\zeta-\frac{1}{2}}\left(K_{0}+\tilde{\delta} \mathcal{K}(t)\right) .
\end{aligned}
$$

Here we have used the fact $\frac{3}{2}\left(\frac{1}{r}-\frac{1}{2}\right)+\frac{1}{2}>1$ since $r<6 / 5$. Similarly, by the estimates (4.43) with $\ell=1$ and $q=2$, and (4.42) -(4.43) with $\ell=0$ and $q=\infty$ of Proposition 4.5 respectively, using the nonlinear estimates (4.44)-(4.46), we deduce

$$
\|\nabla u(t)\|_{L^{2}}+\|\varrho(t)\|_{L^{\infty}}+\|u(t)\|_{L^{\infty}} \lesssim(1+t)^{-\zeta-\frac{1}{2}}\left(K_{0}+\tilde{\delta} \mathcal{K}(t)\right) .
$$

We thus deduce from (4.84)-(4.85) that

$$
\mathcal{H}(t) \lesssim(1+t)^{-\zeta-\frac{1}{2}}\left(K_{0}+\tilde{\delta} \mathcal{K}(t)\right)
$$

Now we substitute (4.86) into (4.81) to obtain

$$
\begin{aligned}
\mathcal{J}^{2}(t) & \lesssim e^{-\lambda t} K_{0}^{2}+\int_{0}^{t} e^{-\lambda(t-\tau)}(1+\tau)^{-2 \zeta-1}\left(K_{0}^{2}+\tilde{\delta}^{2} \mathcal{K}^{2}(t)\right) d \tau . \\
& \lesssim(1+t)^{-2 \zeta-1}\left(K_{0}^{2}+\tilde{\delta}^{2} \mathcal{K}^{2}(t)\right) .
\end{aligned}
$$

By the definition (4.80) of $\mathcal{K}(t)$, we deduce from (4.86)-(4.87) that

$$
\mathcal{K}(t) \lesssim K_{0}+\tilde{\delta} \mathcal{K}(t) .
$$

This implies, since $\tilde{\delta}$ is small,

$$
\mathcal{K}(t) \lesssim K_{0} .
$$

Finally, by the estimates (4.43) with $\ell=0$ and $q=2$ of Proposition 4.5. using the estimates (4.44)-(4.45) with $\ell=0$ and (4.83), in view of (4.80), by (4.89), we obtain

$$
\begin{aligned}
\|u(t)\|_{L^{2}} & \lesssim(1+t)^{-\frac{3}{2}\left(\frac{1}{p}-\frac{1}{2}\right)} K_{0}+\tilde{\delta} \int_{0}^{t}(1+t-\tau)^{-\frac{3}{2}\left(\frac{1}{r}-\frac{1}{2}\right)}(1+\tau)^{-\zeta-\frac{3}{4}} \mathcal{K}(t) d \tau \\
& \lesssim K_{0}(1+t)^{-\zeta}
\end{aligned}
$$


Note that (4.89) implies

$$
\|\varrho(t)\|_{H^{k}}+\|\nabla u(t)\|_{H^{k-1}} \lesssim K_{0}(1+t)^{-\zeta-\frac{1}{2}} .
$$

That is, we have proved (1.9) for $\ell=0$ and (1.10) for $0 \leqslant \ell \leqslant 1$ by the interpolation. To prove the remaining decay estimates in (1.9) -(1.11), we may now employ the arguments used in Case 1. Indeed, since $0<3-3 / r<1$ and $4-3 / r>1$, by the interpolation, we deduce from (4.90)-4.91) that

$$
\begin{aligned}
& \|\varrho(\tau)\|_{L^{2}}\left\|\nabla^{3-\frac{3}{r}} u(\tau)\right\|_{L^{2}}+\|u(\tau)\|_{L^{2}}\left\|\nabla^{4-\frac{3}{r}} u(\tau)\right\|_{L^{2}} \\
& \quad \lesssim K_{0}^{2}(1+\tau)^{-\zeta-\frac{1}{2}}(1+\tau)^{-\zeta-\frac{3-3 / r}{2}}+K_{0}^{2}(1+\tau)^{-\zeta}(1+\tau)^{-\zeta-\frac{1}{2}} \\
& \quad \lesssim K_{0}^{2}(1+\tau)^{-\zeta-\frac{3}{4}} .
\end{aligned}
$$

Here we have used the fact $2 \zeta+1 / 2 \geqslant \zeta+3 / 4$ since $p, r<3 / 2$. So by replacing the estimates (4.67) by the estimates (4.92) and then reproducing the arguments of Case 1, we may derive

$$
\mathcal{N}(t) \lesssim K_{0}+K_{0}^{2}
$$

where $\mathcal{N}(t)$ is defined by (4.64). This in turn together with the interpolation gives (1.9)-(1.11) for $1<r<6 / 5$ by taking $C_{0}=K_{0}+K_{0}^{2}$.

Now in view of these two cases, the proof of Theorem 1.3 is completed.

\section{Appendix A. Analytic tools}

We recall the Sobolev interpolation of the Gagliardo-Nirenberg inequality.

Lemma A.1. Let $2 \leqslant p \leqslant \infty$ and $\alpha, \beta, \gamma \in \mathbb{R}$. Then we have

$$
\left\|\nabla^{\alpha} f\right\|_{L^{p}} \lesssim\left\|\nabla^{\beta} f\right\|_{L^{2}}^{1-\theta}\left\|\nabla^{\gamma} f\right\|_{L^{2}}^{\theta} .
$$

Here $0 \leqslant \theta \leqslant 1$ (if $p=\infty$, then we require that $0<\theta<1$ ) and $\alpha$ satisfy

$$
\alpha+3\left(\frac{1}{2}-\frac{1}{p}\right)=\beta(1-\theta)+\gamma \theta .
$$

Proof. For the case $2 \leqslant p<\infty$, we refer to Lemma 2.4 in [5]; for the case $p=\infty$, we refer to Exercise 6.1.2 in [4].

We then recall the following commutator and product estimates:

Lemma A.2. Let $l \geqslant 0$ and define the commutator

$$
\left[\nabla^{l}, g\right] h=\nabla^{l}(g h)-g \nabla^{l} h
$$

Then we have

$$
\left\|\left[\nabla^{l}, g\right] h\right\|_{L^{p_{0}}} \lesssim\|\nabla g\|_{L^{p_{1}}}\left\|\nabla^{l-1} h\right\|_{L^{p_{2}}}+\left\|\nabla^{l} g\right\|_{L^{p_{3}}}\|h\|_{L^{p_{4}}} .
$$

In addition, we have that for $l \geqslant 0$,

$$
\left\|\nabla^{l}(g h)\right\|_{L^{p_{0}}} \lesssim\|g\|_{L^{p_{1}}}\left\|\nabla^{l} h\right\|_{L^{p_{2}}}+\left\|\nabla^{l} g\right\|_{L^{p_{3}}}\|h\|_{L^{p_{4}}} .
$$

Here $p_{0}, p_{2}, p_{3} \in(1, \infty)$ and

$$
\frac{1}{p_{0}}=\frac{1}{p_{1}}+\frac{1}{p_{2}}=\frac{1}{p_{3}}+\frac{1}{p_{4}} .
$$

Proof. We refer to Lemma 3.1 in [7].

Lastly, we record the estimates of the remainder $\mathcal{R}$ defined by (3.4). 
Lemma A.3. Let $\mathcal{R}$ be defined by (3.4). Then we have that for $l \geqslant 1$,

$$
\left\|\nabla^{l} \mathcal{R}\right\|_{L^{2}} \lesssim \delta\left(\|\nabla \varrho\|_{L^{2}}+\left\|\nabla^{l} \varrho\right\|_{L^{2}}\right)
$$

and

$$
\left\|\nabla^{l} \mathcal{R}\right\|_{L^{2}} \lesssim \delta\left(\|\varrho\|_{L^{\infty}}+\left\|\nabla^{l} \varrho\right\|_{L^{2}}\right) .
$$

Here " $\lesssim "$ stands for " $\leqslant C$ " with the constant $C$ depending on the function $h$, the upper and lower bounds of $\rho_{s}$ and $\left\|\nabla \rho_{s}\right\|_{H^{l}}$.

Proof. We only prove (A.5), while (A.6) can be proved similarly with minor modifications. We may view $\mathcal{R}$ as an operator over $h$, i.e., we define the operator $\mathcal{R}(f)$ of the smooth function $f$ :

$$
\begin{aligned}
\mathcal{R}(f) & :=\int_{\rho_{s}}^{\varrho+\rho_{s}} f^{\prime \prime}(s)\left(\varrho+\rho_{s}-s\right) d s \\
& \equiv \int_{0}^{\varrho} f^{\prime \prime}\left(\varrho+\rho_{s}-\tau\right) \tau d \tau .
\end{aligned}
$$

Then $\mathcal{R}=\mathcal{R}(h)$. It is clear from the definition (A.7) that

$$
\mathcal{R}(f)=O\left(\varrho^{2}\right) .
$$

Moreover, taking the spatial derivative of (A.7) yields

$$
\begin{aligned}
\nabla \mathcal{R}(f) & =f^{\prime \prime}\left(\rho_{s}\right) \varrho \nabla \varrho+\int_{0}^{\varrho} f^{\prime \prime \prime}\left(\varrho+\rho_{s}-\tau\right) \tau d \tau\left(\nabla \varrho+\nabla \rho_{s}\right) \\
& \equiv f^{\prime \prime}\left(\rho_{s}\right) \varrho \nabla \varrho+\mathcal{R}\left(f^{\prime}\right)\left(\nabla \varrho+\nabla \rho_{s}\right) .
\end{aligned}
$$

Hence, by Hölder's and Sobolev's inequalities, we have

$$
\begin{aligned}
\|\nabla \mathcal{R}(f)\|_{L^{2}} & \lesssim\left\|f^{\prime \prime}\left(\rho_{s}\right) \varrho \nabla \varrho\right\|_{L^{2}}+\left\|\mathcal{R}\left(f^{\prime}\right)\left(\nabla \varrho+\nabla \rho_{s}\right)\right\|_{L^{2}} \\
& \lesssim\left\|f^{\prime \prime}\left(\rho_{s}\right)\right\|_{L^{\infty}}\|\varrho\|_{L^{\infty}}\|\nabla \varrho\|_{L^{2}}+\|\varrho\|_{L^{\infty}}\|\varrho\|_{L^{6}}\left(\|\nabla \varrho\|_{L^{3}}+\left\|\nabla \rho_{s}\right\|_{L^{3}}\right) \\
& \lesssim\|\varrho\|_{L^{\infty}}\|\nabla \varrho\|_{L^{2}} \lesssim \delta\|\nabla \varrho\|_{L^{2}} .
\end{aligned}
$$

Since $\mathcal{R}=\mathcal{R}(h)$, we deduce A.5 for $l=1$.

Now for $l \geqslant 2$, by the identity (A.9) and the product estimates (A.4) of Lemma A.2, we obtain

$$
\begin{aligned}
\left\|\nabla^{l} \mathcal{R}(f)\right\|_{L^{2}}=\left\|\nabla^{l-1}(\nabla \mathcal{R}(f))\right\|_{L^{2}} \\
\lesssim\left\|\nabla^{l-1}\left(f^{\prime \prime}\left(\rho_{s}\right) \varrho \nabla \varrho\right)\right\|_{L^{2}}+\left\|\nabla^{l-1}\left(\mathcal{R}\left(f^{\prime}\right)\left(\nabla \varrho+\nabla \rho_{s}\right)\right)\right\|_{L^{2}} \\
\lesssim\left\|\nabla^{l-1}\left(f^{\prime \prime}\left(\rho_{s}\right)\right)\right\|_{L^{3}}\|\varrho \nabla \varrho\|_{L^{6}}+\left\|f^{\prime \prime}\left(\rho_{s}\right)\right\|_{L^{\infty}}\left\|\nabla^{l-1}(\varrho \nabla \varrho)\right\|_{L^{2}} \\
\quad+\left\|\mathcal{R}\left(f^{\prime}\right)\right\|_{L^{\infty}}\left\|\nabla^{l} \varrho\right\|_{L^{2}}+\left\|\mathcal{R}\left(f^{\prime}\right)\right\|_{L^{6}}\left\|\nabla^{l} \rho_{s}\right\|_{L^{3}} \\
\quad+\left(\|\nabla \varrho\|_{L^{\infty}}+\left\|\nabla \rho_{s}\right\|_{L^{\infty}}\right)\left\|\nabla^{l-1} \mathcal{R}\left(f^{\prime}\right)\right\|_{L^{2}} \\
\quad \lesssim\|\varrho\|_{L^{6}}\|\nabla \varrho\|_{L^{\infty}}+\|\varrho\|_{L^{\infty}}\left\|\nabla^{l} \varrho\right\|_{L^{2}}+\left\|\nabla^{l-1} \varrho\right\|_{L^{6}}\|\nabla \varrho\|_{L^{3}} \\
\quad+\|\varrho\|_{L^{\infty}}^{2}\left\|\nabla^{l} \varrho\right\|_{L^{2}}+\|\varrho\|_{L^{\infty}}\|\varrho\|_{L^{6}}+\left\|\nabla^{l-1} \mathcal{R}\left(f^{\prime}\right)\right\|_{L^{2}} \\
\lesssim \delta\left(\|\nabla \varrho\|_{L^{2}}+\left\|\nabla^{l} \varrho\right\|_{L^{2}}\right)+\left\|\nabla^{l-1} \mathcal{R}\left(f^{\prime}\right)\right\|_{L^{2}} .
\end{aligned}
$$

By this recursive inequality (A.11), we obtain that for $l \geqslant 2$,

$$
\begin{aligned}
\left\|\nabla^{l} \mathcal{R}\right\|_{L^{2}} \equiv\left\|\nabla^{l} \mathcal{R}(h)\right\|_{L^{2}} & \lesssim \delta \sum_{\ell=1}^{l}\left\|\nabla^{\ell} \varrho\right\|_{L^{2}}+\left\|\nabla \mathcal{R}\left(h^{(l-1)}\right)\right\|_{L^{2}} \\
& \lesssim \delta\left(\|\nabla \varrho\|_{L^{2}}+\left\|\nabla^{l} \varrho\right\|_{L^{2}}\right) .
\end{aligned}
$$


Here in the last inequality we have used the inequality (A.10) for $f=h^{(l-1)}$. This proves A.5 for $l \geqslant 2$, and the proof of the lemma is completed.

\section{ACKNOWLEDGEMENTS}

The authors are deeply grateful to the referees for the valuable comments and suggestions.

\section{REFERENCES}

[1] R. J. Duan, H. X. Liu, S. Ukai And T. YAng, Optimal $L^{p}-L^{q}$ convergence rate for the compressible Navier-Stokes equations with potential force, J. Differential Equations, 238 (2007), pp. 220-233.

[2] R. J. Duan, S. Ukai, T. YAng And H. J. ZhaO, Optimal convergence rate for the compressible NavierStokes equations with potential force, Math. Models Methods Appl. Sci., 17 (2007), pp. 737-758.

[3] E. Feireisl, A. Novotný And H. Petleltová, On the existence of globally defined weak solutions to the Navier-Stokes equations of isentropic compressible fluids, J. Math. Fluid Mech., 3 (2001), pp. 358-392.

[4] L. Grafakos, Classical and Modern Fourier Analysis, Pearson Education, Inc., Prentice Hall, 2004.

[5] Y. Guo, Y. J. Wang, Decay of dissipative equations and negative Sobolev spaces, Comm. Partial Differential Equations, 37 (2012), pp. 2165-2208.

[6] L. HsiaO, Q. C. Ju AND S. WANG, The asymptotic behavior of global smooth solutions to the multidimensional hydrodynamic model for semiconductors, Math. Meth. Appl. Sci., 26 (2003), pp. 1187-1210.

[7] N. Ju, Existence and uniqueness of the solution to the dissipative 2D Quasi-Geostrophic equations in the Sobolev space, Commun. Math. Phys., 251 (2004), pp. 365-376.

[8] T. Kobayashi, T. Suzuki, Weak solutions to the Navier-Stokes-Poisson equation, Adv. Math. Sci. Appl., 18 (2008), pp. 141-168.

[9] P. L. Lions, Mathematical Topics in Fluids Mechanics, Oxford Lecture Ser. Math. Appl., vol. 2, Clarendon Press, Oxford University Press, New York, 1998.

[10] H. L. Li, A. Matsumura and G. J. Zhang, Optimal decay rate of the compressible Navier-Stokes-Poisson system in $\mathbb{R}^{3}$, Arch. Rational Mech. Anal., 196 (2010), pp. 681-713.

[11] A. Matsumura, T. Nishida, The initial value problems for the equations of motion of viscous and heatconductive gases, J. Math. Kyoto Univ., 20 (1980), pp. 67-104.

[12] A. Matsumura, T. Nishida, The initial value problem for the equations of motion of compressible viscous and heat-conductive fluids, Proc. Japan Acad. Ser. A, 55 (1979), pp. 337-342.

[13] G. Ponce, Global existence of small solution to a class of nonlinear evolution equations, Nonlinear Anal., 9 (1985), pp. 339-418.

[14] E. M. Stein, Singular Integrals and Differentiability Properties of Functions, Princeton University Press, 1970 .

[15] Z. TAN, Y. J. WANG, Global existence and large-time behavior of weak solutions to the compressible magnetohydrodynamic equations with Coulomb force, Nonlinear Anal., 71 (2009), pp. 5866-5884.

[16] Y. J. WAng, Decay of the Navier-Stokes-Poisson equations, J. Differential Equations, 253 (2012), pp. 273297.

School of Mathematical Sciences, Xiamen University, Xiamen, Fujian 361005, China

E-mail address, Z. Tan: ztan85@163.com

School of Mathematical Sciences, Xiamen University, Xiamen, Fujian 361005, China

E-mail address, Y. J. Wang: yanjin_wang@xmu.edu.cn

School of Mathematical Sciences, Xiamen University, Xiamen, Fujian 361005, China

E-mail address, Y. Wang: wangyongxmu@163.com 